NATIONAL AERONAUTICS AND SPACE ADMINISTRATION WP

Technical Report 32-1483

\title{
Plasma Properties and Performance of Mercury Ion Thrusters
}

\section{T. D. Masek}
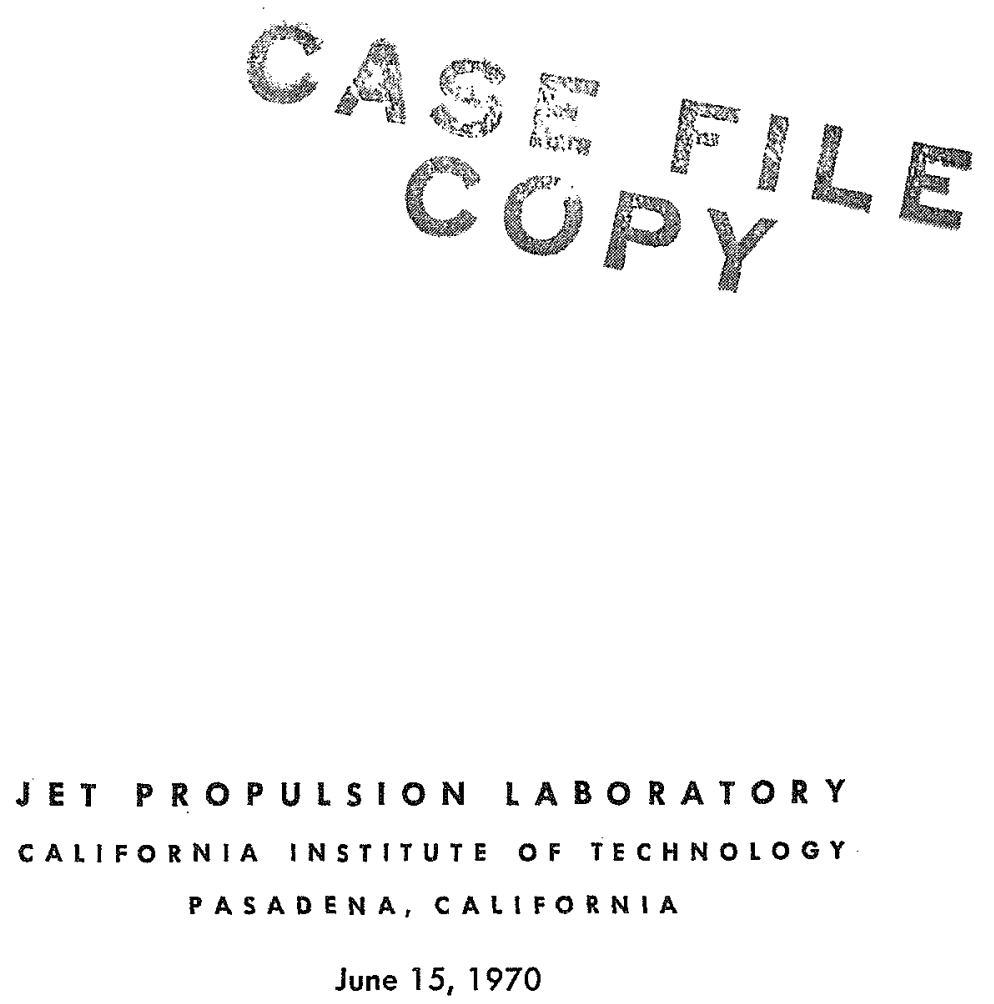
NATIONAL AERONAUTICS AND SPACE ADMINISTRATION

Technical Report 32-1483

\title{
Plasma Properties and Performance of Mercury lon Thrusters
}

\author{
T. D. Masek
}

SET PROPLSION LABORATORY CALIFORNIA INSTITUTE OF TECHNOLOGY PASADENA, CALIFORNIA June 15,1970 
Prepared Under Contract No. NAS 7-100 National Aeronautics and Space Administration 


\section{Acknowledgment}

The author acknowledges the valuable assistance of the Applications Studies Group Laboratory Staff in making the experimental measurements, and the helpful suggestions and critical review of the paper by Daniel J. Kerrisk of JPL. 



\section{Contents}

I. Introduction ...................... 1

II. Fundamentals of Operation . . . . . . . . . . . . . . . . . 2

A. General Operation . . . . . . . . . . . . . . . . . . 2

B. Ion Fluxes. . . . . . . . . . . . . . . . . . . . . . . . . 3

1. Beam flux. . . . . . . . . . . . . . . . . 3

2. Wall flux. . . . . . . . . . . . . . . . 10

C. Electron Flux. . . . . . . . . . . . . . . . . . . . . . . . 10

D. Ion Production Rate . . . . . . . . . . . . . . . . . . . . . 11

E. Ion Production Cost . . . . . . . . . . . . . . . . . . . . . 13

F. Discussion . . . . . . . . . . . . . . . . . . . . 16

III. Effects of Thruster Configuration . . . . . . . . . . . . . . 17

A. Pole Pieces . . . . . . . . . . . . . . . . . . . . . . . . 17

B. Cathode Position . . . . . . . . . . . . . . . . . . . . . . 18

C. Screen Grid . . . . . . . . . . . . . . . . . . . . . . . 19

D. Propellant Introduction . . . . . . . . . . . . . . . . . . . 19

IV. Effects of Operating Conditions . . . . . . . . . . . . . . . . . 20
A. Propellant Flowrate . . . . . . . . . . . . . . . . . . . 20
B. Propellant Utilization Efficiency . . . . . . . . . . . . . . . . . 21
C. Discharge Voltage . . . . . . . . . . . . . . . . . . . . 23
D. Magnet Current. . . . . . . . . . . . . . . . . . . . . . . 24

v. Conclusions . . . . . . . . . . . . . . . . . . . . . . . . 25

Appendix. Modified Bohm Criterion . . . . . . . . . . . . . . . . 26

Nomenclature . . . . . . . . . . . . . . . . . 27

References. . . . . . . . . . . . . . . . . . 27

\section{Tables}

1. Comparison of ion flux from Bohm criteria with beam current . . . . . . . 10

2. Summary of ion wall-flux calculations . . . . . . . . . . . . . . . 11

3. Summary of ion production rate calculations . . . . . . . . . . . . . 13

4. Electron energy losses. . . . . . . . . . . . . . . . . . . . . 17 


\section{Contents (contd)}

\section{Figures}

1. Thruster and plasma schematic diagram . . . . . . . . . . . . . . 2

2. Plasma properties in the 15 -cm-diameter thruster-flowrate, $4.7 \mathrm{~g} / \mathrm{h}$; propellant utilization efficiency, $77 \%$. . . . . . . . . . . . . . . . . . 4

3. Plasma properties in the 15 -cm-diameter thruster-flowrate, $4.7 \mathrm{~g} / \mathrm{h}$;

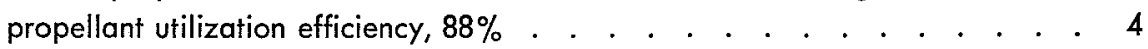

4. Plasma properties in the 20 -cm-diameter thruster-flowrate, $5.7 \mathrm{~g} / \mathrm{h}$; propellant utilization efficiency, $89 \%$. . . . . . . . . . . . . . . . . 5

5. Plasma properties in the 20 -cm-diameter thruster-flowrate, $6.9 \mathrm{~g} / \mathrm{h}$; propellant utilization efficiency, $90 \%$. . . . . . . . . . . . . . . 6

6. Plasma properties in the 20 -cm-diameter thruster-flowrate, $9.0 \mathrm{~g} / \mathrm{h}$; propellant utilization efficiency, $88 \%$. . . . . . . . . . . . . . . . 7

7. Plasma properties in the 20 -cm-diameter thruster-flowrate, $9.2 \mathrm{~g} / \mathrm{h}$;

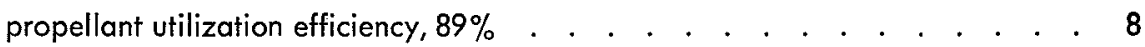

8. Plasma sheath screen grid boundary configurations . . . . . . . . . . . . 9

9. Configuration for neutral atom density analysis . . . . . . . . . . . . 12

10. Ratio of atom density at a given position to that at the center grid position . . 13

11. Local ion production rate by Maxwellian and primary electrons . . . . . . 14

12. Local ion production rate by primary electrons . . . . . . . . . . . . 15

13. Local collisional energy loss factor . . . . . . . . . . . . . . . 16

14. Cathode and pole piece configurations . . . . . . . . . . . . . . 17

15. Ion density variation with propellant flowrate . . . . . . . . . . . . 20

16. Ratio of primary electron density to ion density for four flowrates . . . . . . 21

17. Typical discharge loss-propellant utilization efficiency curve . . . . . . . 21

18. Ion density variation with propellant utilization efficiency . . . . . . . . 21

19. Ion density radial variation as a function of propellant utilization efficiency . . . . . . . . . . . . . . . . . . . . . . . 22

20. Maxwellian electron temperature variation with propellant utilization efficiency . . . . . . . . . . . . . . . . . . . . . 22

21. Maxwellian electron temperature radial variation as a function of propellant utilization efficiency . . . . . . . . . . . . . . . 23

22. Ion density and Maxwellian electron temperature variation with discharge voltage . . . . . . . . . . . . . . . . . . 24

23. Ion density and Maxwellian electron temperature variation with magnet current . . . . . . . . . . . . . . . . . . . . . 24 


\begin{abstract}
The objectives of this report are (1) to present a description of the electron bombardment ion thruster operation and show the relationship of the plasma to this operation, (2) to show a method for computing the discharge power per beam ion from the plasma properties for comparison with the measured value, (3) to interpret the variations of discharge power per beam ion with variations in operating conditions in terms of the plasma properties, and (4) to interpret the results of recent thruster improvement studies in terms of the effect of thruster configuration on the plasma properties.
\end{abstract}

Langmuir probe measurements in conventional 15- and 20-cm-diameter thrusters using mercury are presented. The 15-cm-diameter thruster, of 1962 vintage, was operated at high flowrates $(650 \mathrm{~mA}$ equivalent mercury flowrate) for comparison with previous lower flowrate data and to establish reference thruster plasma characteristics. Measurements made in an improved $20-\mathrm{cm}$-diameter thruster are used to show the effects of operating conditions on the plasma and for comparison with the reference thruster characteristics.

A modified form of the Bohm stable sheath criterion is shown to apply for computing ion fluxes. The use of this criterion, along with calculations of ion production rates and electron fluxes, permits a more accurate and comprehensive picture of discharge losses than has been obtained previously. 


\section{Plasma Properties and Performance of Mercury Ion Thrusters}

\section{Introduction}

Quantitative or analytical relationships between the plasma properties and operation of mercury thrusters have been of interest for some time (Refs. 1, 2). Previous studies have indicated the difficulty of obtaining useful analytical expressions describing overall thruster operation (Refs. 2, 3). To obtain analytical solutions requires, in general, a great number of assumptions because of the complex nature of the plasma. The non-Maxwellian electron velocity distribution function in mercury, nonuniform applied magnetic fields, an unknown neutral atom density distribution, and general mathematical difficulties all severely restrict the validity, and, hence, the usefulness of analytical solutions. On the other hand, experimental studies of plasma properties (Refs. 4,5), while providing quantitative data at specific operating points, have not heretofore provided the desired relationships between these properties and thruster operating parameters.

This report presents a description of thruster operation, based on plasma measurements, that is consistent with gross thruster measurements (i.e., beam current and discharge losses). A method is described for computing ion beam current, discharge losses, and propellant utilization efficiency from measurements of ion density and electron temperature. As a part of these calculations, the ion flux to the discharge chamber walls, the electron current to the anode, and the ion production rate are found.

Using this description, recent thruster efficiency improvements are explained (Refs. 6, 7). These improvements, achieved through thruster configuration changes, can be traced to changes in the plasma characteristics. A comparison of the ratio of beam-to-wall ion fluxes and the ion production costs in old and improved thrusters is made. This comparison allows the basic differences in operation to be compared with configuration differences. In addition, the effects of operating conditions (propellant utilization, propellant flowrate, discharge voltage, and magnet current) on discharge losses reported in Ref. 7 are analyzed.

The data required for this study were obtained with movable Langmuir probes in 15- and 20-cm-diameter thrusters. Data published previously (Ref. 8), as well as new measurements, are presented for the "unimproved" 15-cm-diameter thruster. All "improved" thruster data were obtained with a 20-cm-diameter thruster. Experimental setups have been reported previously (Refs. 8, 9) for both thrusters and are not described herein. 


\section{Fundamentals of Operation}

\section{A. General Operation}

A description of the general features of this type of thruster is required to discuss the mechanisms controlling bombardment thruster operation in detail. Figure 1 illustrates the processes involved in producing and accelerating ions.

Electrons are accelerated away from the cathode by an electric field (produced by the discharge voltage) and collide with atoms, ions, and other electrons. The resulting low-pressure discharge (approximately $10^{-3}$ torr) would ordinarily allow long mean free paths for electrons. However, an axial magnetic field is provided to restrict the radial motion of the electrons. This field gives electrons a long cycloidal path with a cyclotron radius of the order of $1 \mathrm{~cm}$. Electrons are inhibited from reaching the walls, which are held at cathode potential, by the wall sheath. Because they spiral around the field lines, electrons require collisions to obtain a radial drift velocity. The ionization process depends upon the electron energy, electron density, and atom density. The mean energy of an electron depends on both the electron density and the

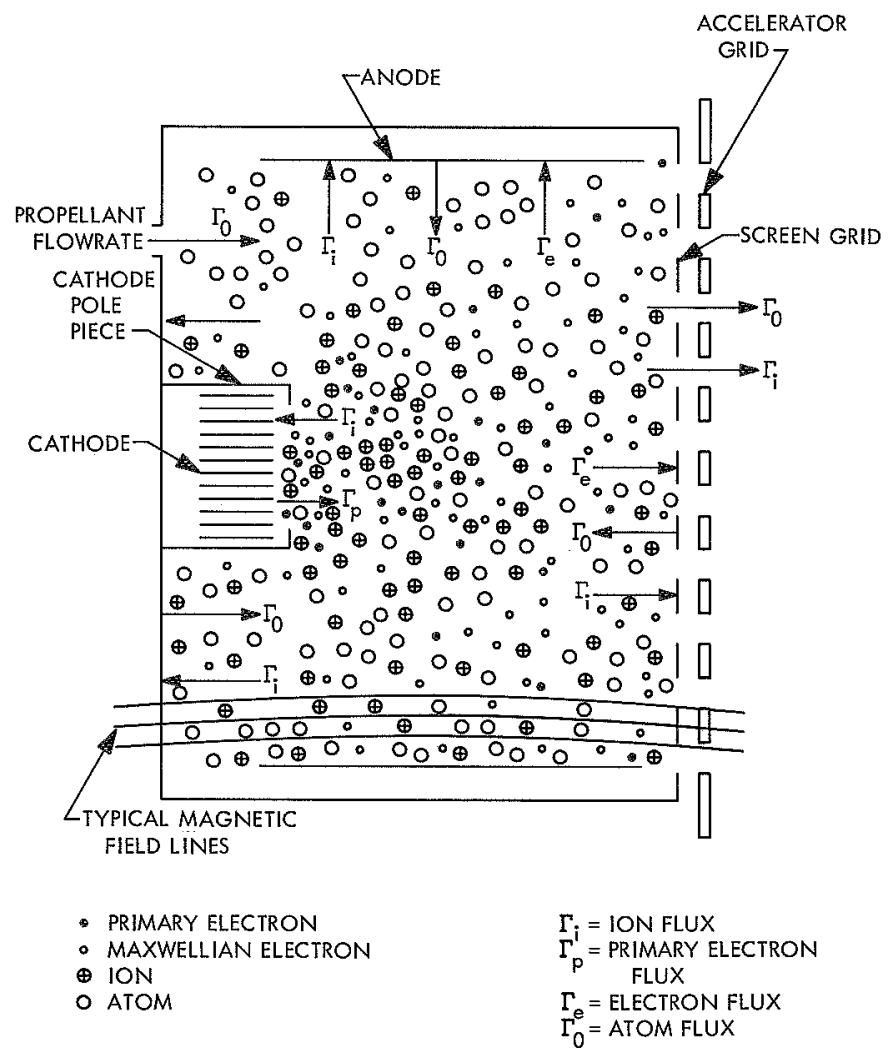

Fig. 1. Thruster and plasma schematic diagram discharge voltage and is a major factor in efficient thruster operation. The factors controlling electron energy, or more specifically the electron velocity distribution function, are basic to the plasma.

As in all low-pressure plasmas of this type with relatively large anodes, the plasma potential is generally positive with respect to the anode (Ref. 10). Since this potential also appears at the cathode sheath, electrons initially enter the plasma with energies slightly higher than the discharge voltage ( 35 to $45 \mathrm{~V}$ for conventional mercury thrusters).

These initial electrons, primary electrons, are shown as black dots in Fig. 1. Collisions between electrons, or inelastic collisions of primary electrons with atoms or ions, redistribute the primary electron energy. Electrons produced in ionization or "thermalized" primary electrons can be considered to form a second distinct group (small open circles in Fig. 1). This group in general has a Maxwellian distribution function, although variations from Maxwellian have been observed (Ref. 5). A combination of these two groups is commonly found in the bombardment thruster. Although the primary electron density is low (less than $10 \%$ of the total electron density), the primary electrons usually contribute approximately half of the ionization.

Ionization occurs for the most part in a one-step process from the atomic ground state. Ionization from excited states is relatively infrequent because transition times (Ref. 11) (approximately $10^{-7} \mathrm{sec}$ ) are much shorter than electron-atom collision times (Ref. 2) (approximately $5 \times 10^{-5} \mathrm{sec}$ for $30-\mathrm{eV}$ primary electrons and a neutral density of $\left.10^{12} \mathrm{~cm}^{-3}\right)$.

Ion motion is determined primarily by the plasma potential. This can be verified by considering ion collisions with electrons, ions, and atoms. For collisions with electrons when the electron speed is much higher than the ion speed, the momentum exchange per collision, divided by the ion momentum, is given approximately by (Ref. 12)

$$
\frac{\Delta\left(m_{i} v_{i}\right)}{m_{i} v_{i}}=\left(\frac{m_{e} \epsilon_{e}}{m_{i} \epsilon_{i}}\right)^{1 / 2}
$$

where $m$ is the particle mass, $\epsilon$ the energy, $v$ the speed, and the subscripts $i$ and $e$ refer to ions and electrons, respectively. Thus, for typical mercury conditions $\epsilon_{e} / \epsilon_{i}=100$, $m_{e} / m_{i}=2.7 \times 10^{-6}$, the ion momentum changes only approximately $1.6 \%$ per electron collision. Although up 
to approximately 50 collisions occur per centimeter of ion path length (Ref. 2), the net force applied to the ion by these electron impacts should be small because of the randomness of the electron velocities. Therefore, the ion motion is essentially unaffected by collisions with electrons.

The largest cross section for ion-atom collisions is that for charge exchange. For an ion speed of $3 \times 10^{4} \mathrm{~cm} / \mathrm{s}$, typical of thermal ions, this cross section is approximately $2 \times 10^{-14} \mathrm{~cm}^{2}$ (Ref. 13). With a typical atom density of $10^{12} \mathrm{~cm}^{-3}$, the mean free path is $50 \mathrm{~cm}$ and is much greater than ordinary thruster dimensions.

Ion-ion collisions are not important in this situation because all ions at a given position are moving together. Therefore, with collisions having a small effect on ion motion, ions are accelerated in the general direction of the maximum plasma potential gradient. A portion of the ions flow toward the accelerating grids, cross the plasma sheath at the screen grid, and accelerate through several kilovolts. The remaining ion flux goes to the anode, housing, screen grid, and cathode.

Ions reaching the walls recombine to form atoms, which evaporate, along with the atoms reaching the surfaces directly, to form a "virtual" propellant source. The evaporating atoms have velocities corresponding to typical wall temperatures of 150 to $250^{\circ} \mathrm{C}$. The magnitude of this source will be shown to be greater than the main propellant flowrate.

The cost of producing ions $\epsilon_{t}$, determined by dividing the discharge power by the beam current, can be expressed as the product of two factors:

$$
\epsilon_{t}=\omega \epsilon_{b}, \quad \mathrm{eV} / \text { beam ion }
$$

where $\epsilon_{b}$ is the basic cost of producing ions in the plasma and $\omega$ is the ratio of total ion flux (wall flux plus beam flux) to beam ion flux. Both $\epsilon_{b}$ and $\omega$ depend on the plasma characteristics and thruster configuration. Calculations of $\omega$ and $\epsilon_{b}$ are presented in the following sections.

\section{B. Ion Fluxes}

Since plasma ion motion is determined almost entirely by the plasma potential distribution, the ion fluxes cannot be computed by usual diffusion theory methods. However, a direct method is available through use of the Bohm stable sheath criterion (Ref. 14). This criterion establishes the minimum ion energy, normal to the sheath, necessary to form a stable sheath. In normal thruster operation the sheaths are stable and the Bohm criterion can be expected to apply. In the case of a Maxwellian electron distribution, this minimum energy was shown to be $k T_{e} / 2 e$ (i.e., half the electron temperature at the sheath). The presence of primary electrons modifies this ion energy slightly. As shown in the Appendix, the minimum possible ion energy at the sheath, when primary electrons are present, is

$$
\epsilon_{i}=\frac{k T_{e}}{2 e}\left(\frac{n_{i}}{n_{m}}\right)
$$

where $n_{m}$ is the Maxwellian electron density, $n_{i}$ the ion density, and $T_{e}$ the temperature of the Maxwellian electrons. In the present calculations, the density ratio in Eq. (3) differs significantly from unity only at the screen grid and cathode.

To verify the validity of the criterion given in Eq. (3), the ion flux through the screen grid computed from plasma properties can be compared with the measured ion beam current.

1. Beam flux. By using Eq. (3), the beam current can be written as

$$
I_{b}=2 \pi e \phi_{i} \int_{0}^{r} n_{i}(r, L) v_{i}(r, L) r d r
$$

where

$$
v_{i}(r, L)=\left[\frac{k T_{e}(r, L)}{m_{i}}\left(\frac{n_{i}(r, L)}{n_{m}(r, L)}\right)\right]^{1 / 2}
$$

The term $\phi_{i}$ accounts for screen grid blockage and $n_{i}(r, L)$ is the ion density at the screen grid. The integration can be replaced with a summation to obtain

$$
I_{b}=e \phi_{i} \sum_{j}\left(n_{i} v_{i}\right)_{j} A_{j}
$$

where $A_{j}$ is a concentric segment of grid area. This approximation by a summation is consistent with the possible experimental errors in $n_{i}$ and $v_{i}$.

Typical distributions of Maxwellian and primary electron density, plasma potential, primary electron energy, and Maxwellian electron temperature, obtained with Langmuir probes, are shown for the 15 -cm-diameter (Figs. 2 and 3) and 20-cm-diameter (Figs. 4 to 7) thrusters. In the 15-cm-diameter thruster, the electron energy distribution was entirely Maxwellian within experimental error. This is attributed to the high plasma density opera- 
tion needed to obtain beam current densities equivalent to the present 20-cm-diameter thruster (approximately $3 \mathrm{~mA} / \mathrm{cm}^{2}$. The ratio of primary electron density to ion density in the $20-\mathrm{cm}$-diameter thruster is relatively uni-
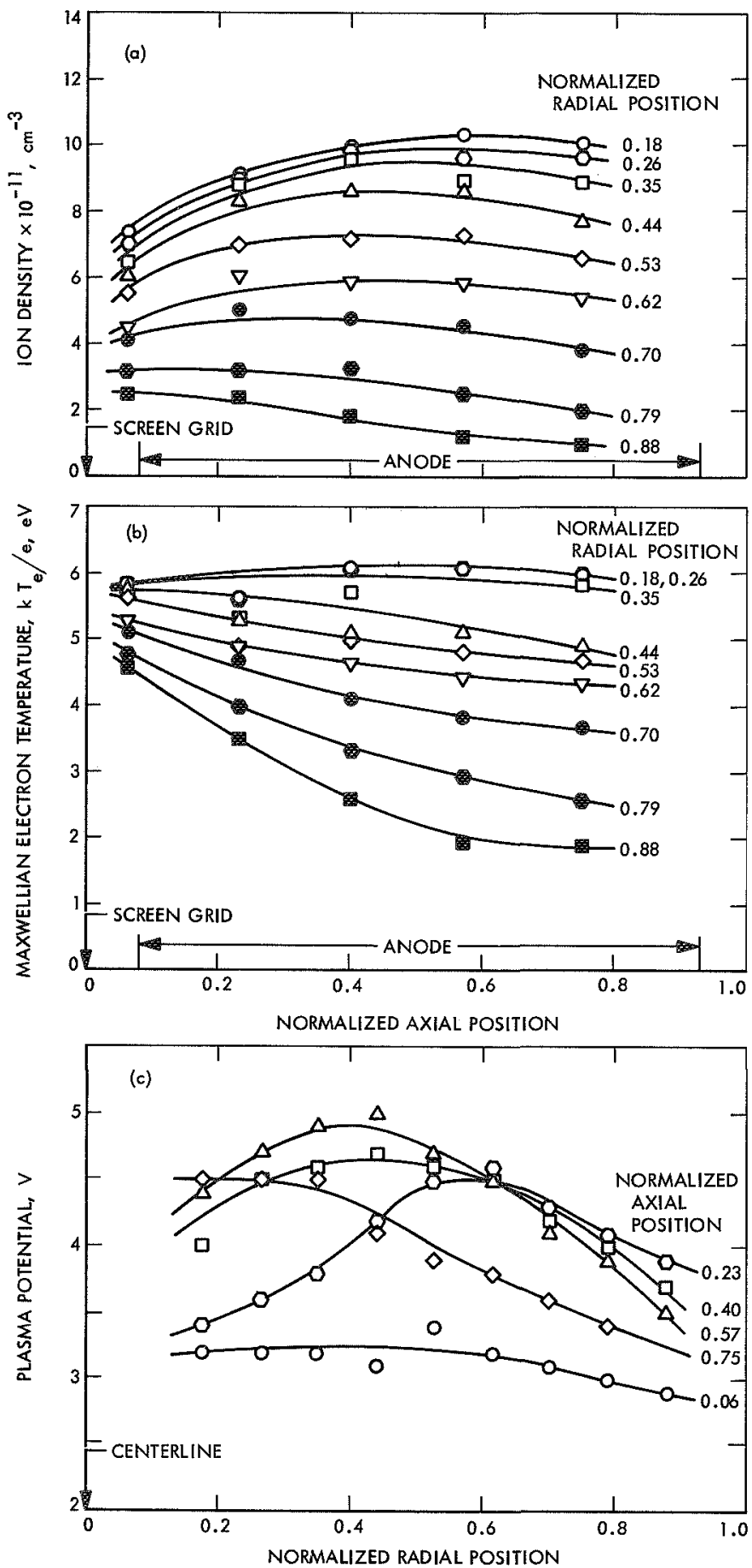

Fig. 2. Plasma properties in the $15-\mathrm{cm}$-diameter thruster -flowrate, $4.7 \mathrm{~g} / \mathrm{h}$; propellant utilization efficiency, $77 \%$ form at the screen grid. Therefore, an average value of $n_{i} / n_{m}$ was used in the calculations. It should be noted that, in the 20-cm-diameter thruster, the cathode was mounted on a "pole piece" (Fig. 1). This element was not used in the $15-\mathrm{cm}$-diameter thruster.
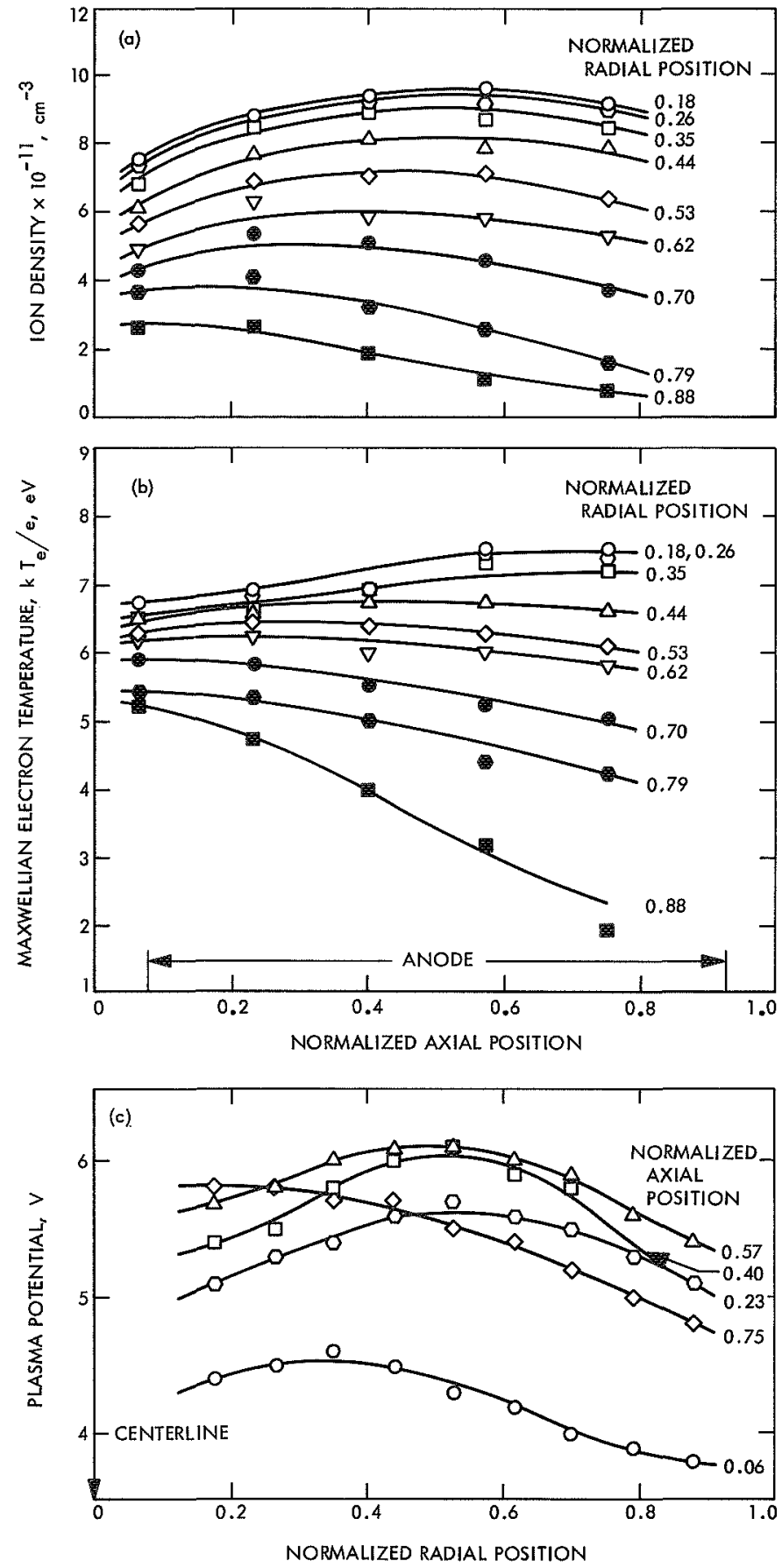

Fig. 3. Plasma properties in the $15-\mathrm{cm}$-diameter thruster -flowrate, $4.7 \mathrm{~g} / \mathrm{h}$; propellant utilization efficiency, $88 \%$ 

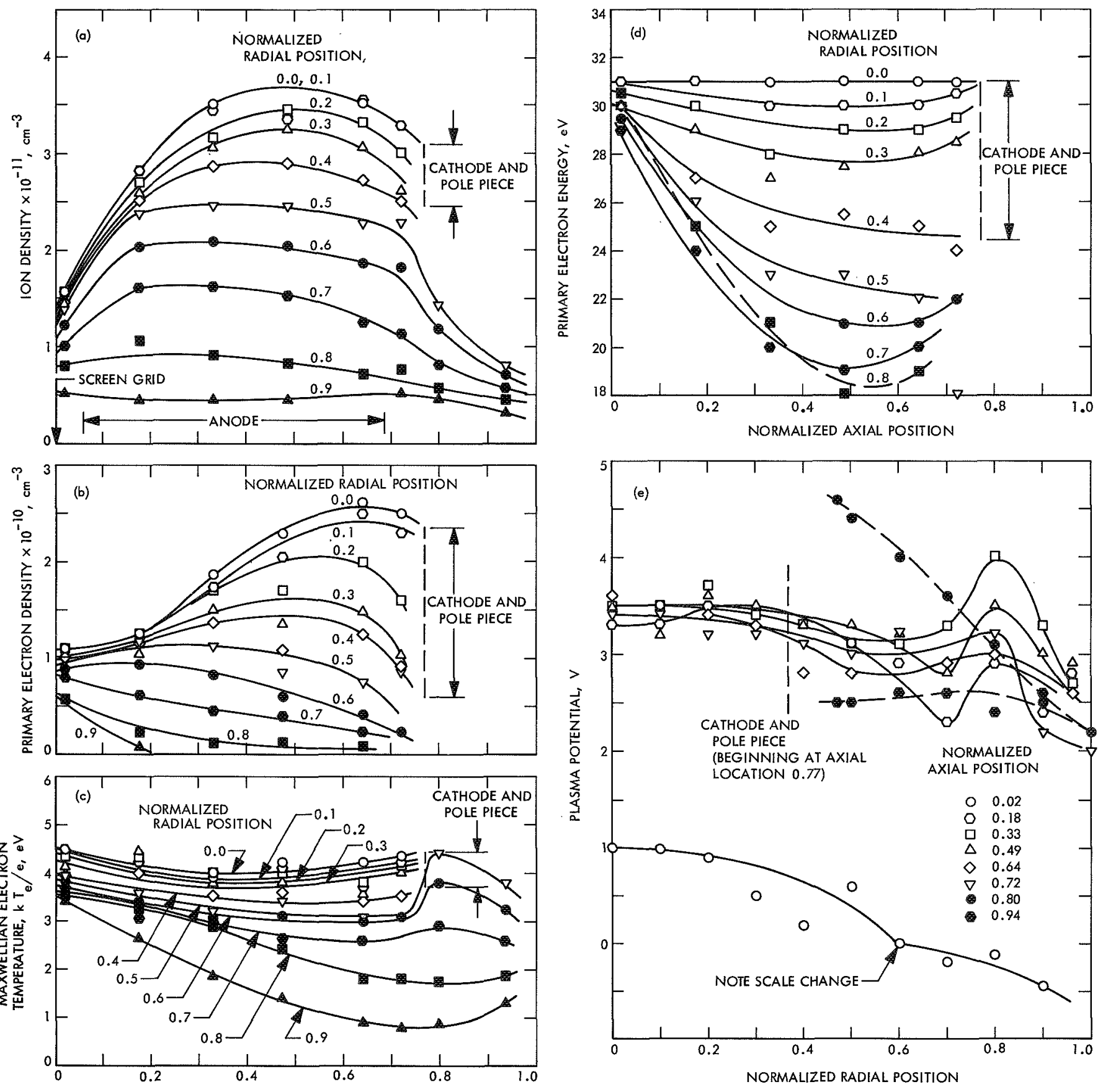

Fig. 4. Plasma properties in the 20 -cm-diameter thruster-flowrate, $5.7 \mathrm{~g} / \mathrm{h}$; propellant utilization efficiency, $89 \%$ 

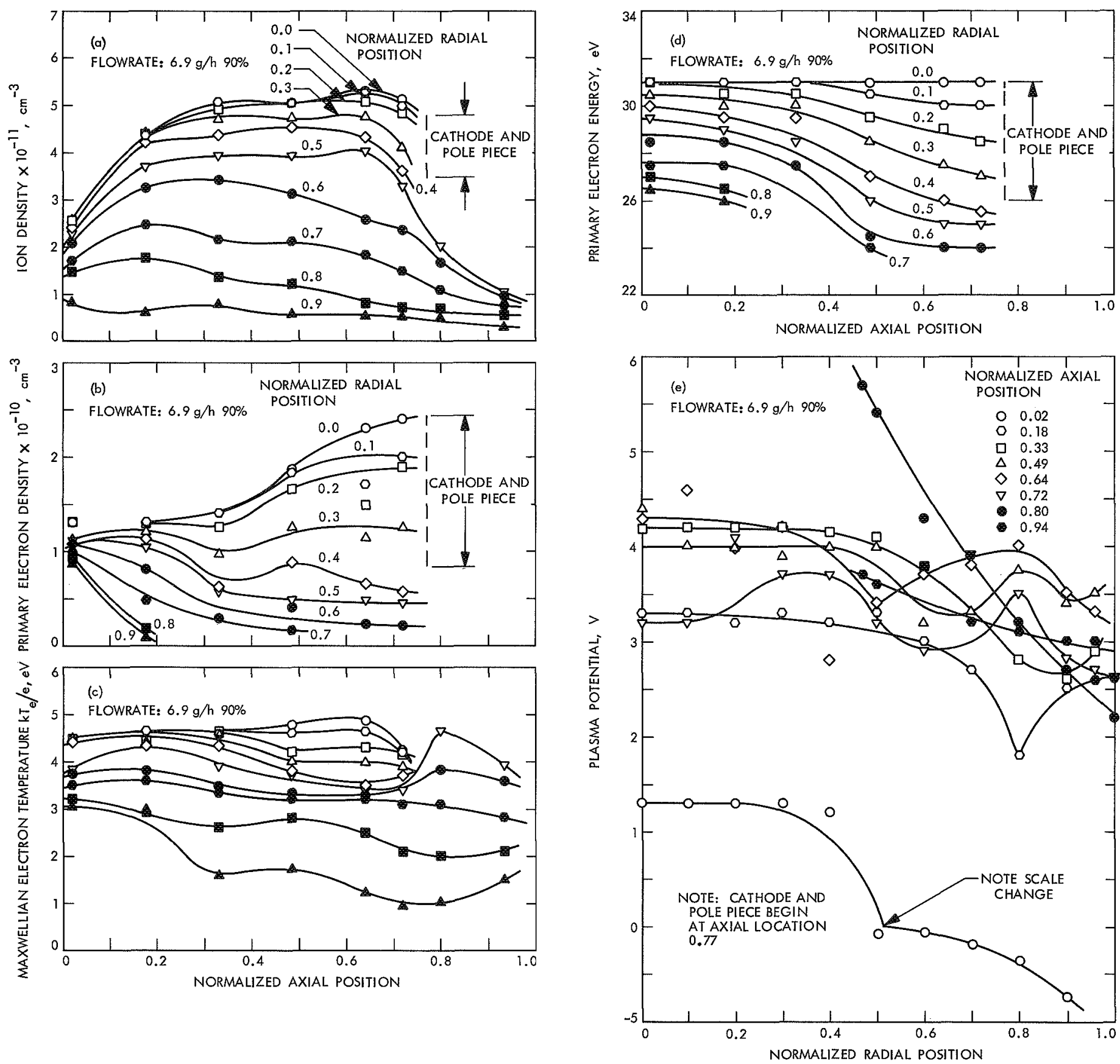

Fig. 5. Plasma properties in the $20-\mathrm{cm}$-diameter thruster-flowrate, $6.9 \mathrm{~g} / \mathrm{h}$; propellant utilization efficiency, $90 \%$ 

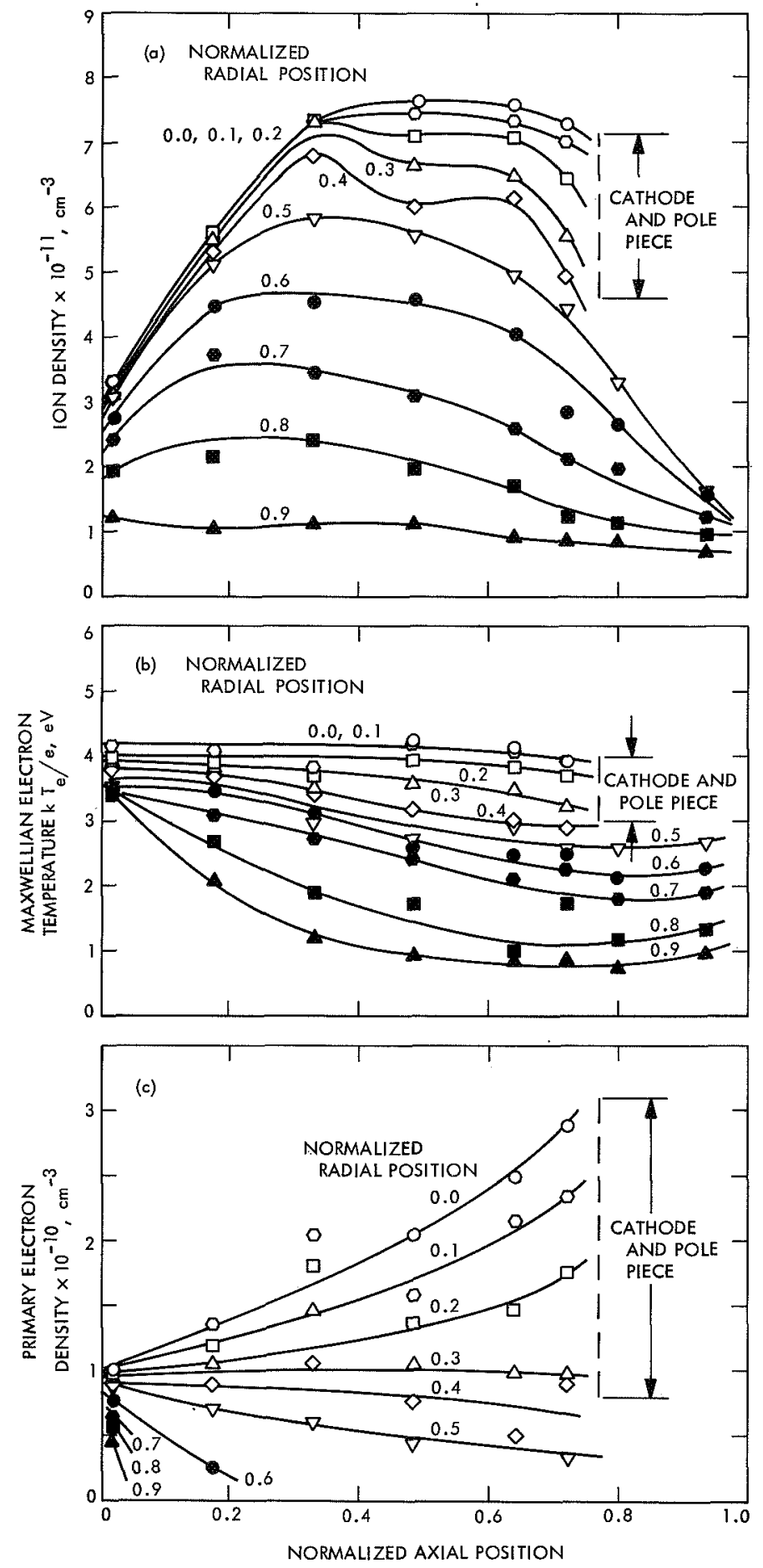
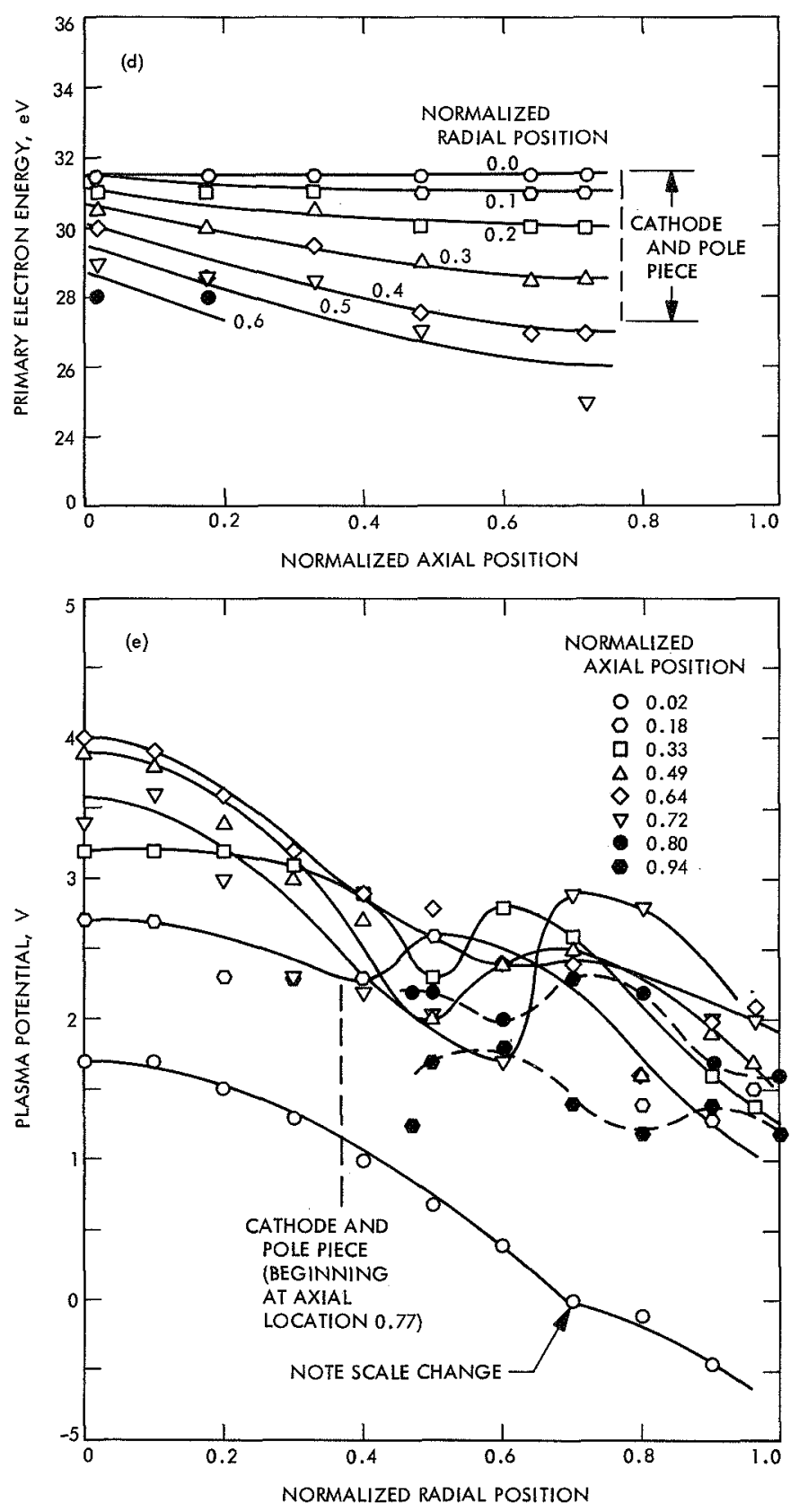

Fig. 6. Plasma properties in the 20 - $\mathrm{cm}$-diameter thruster-flowrate, $9.0 \mathrm{~g} / \mathrm{h}$; propellant utilization efficiency, $88 \%$ 

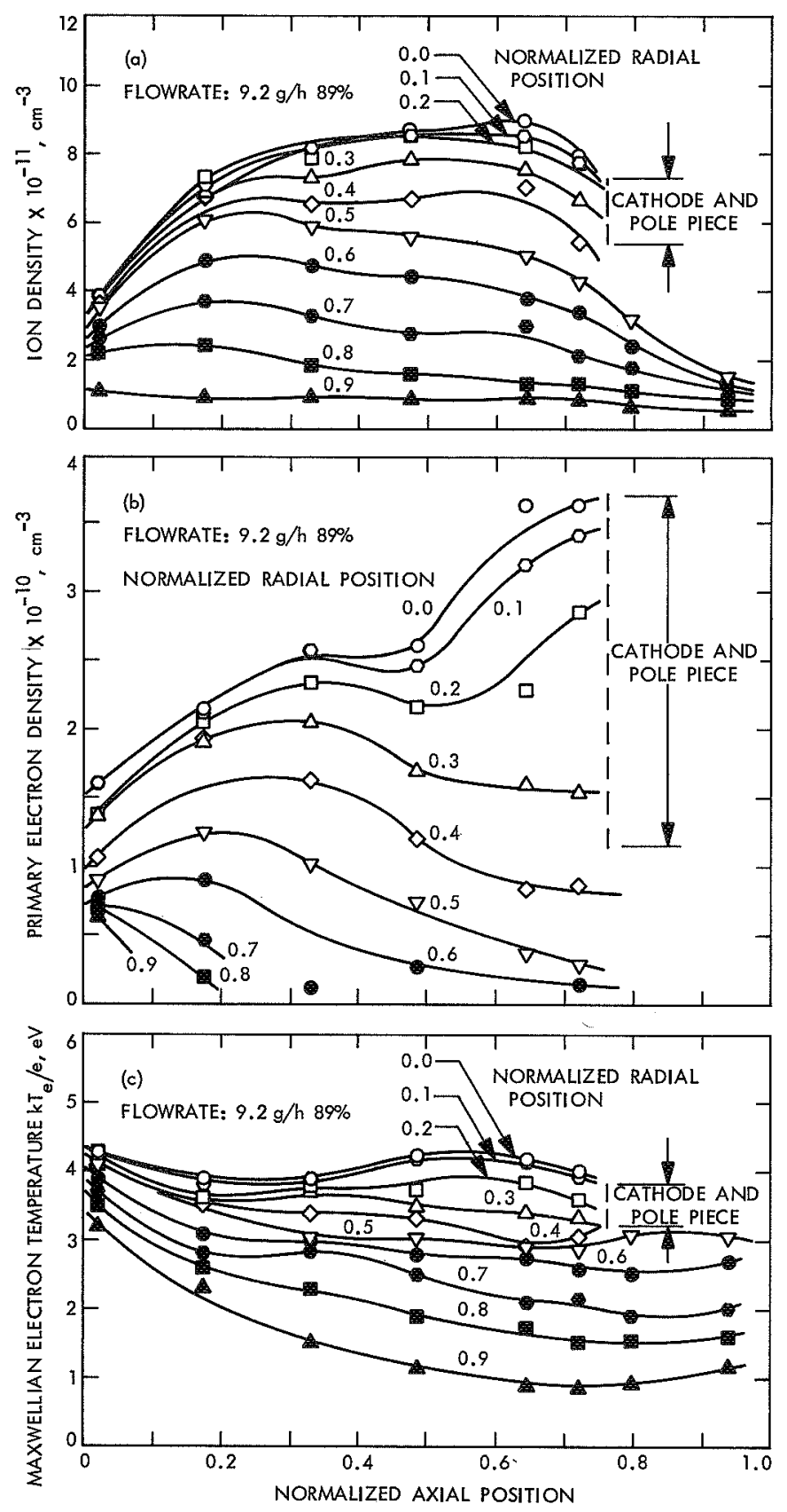
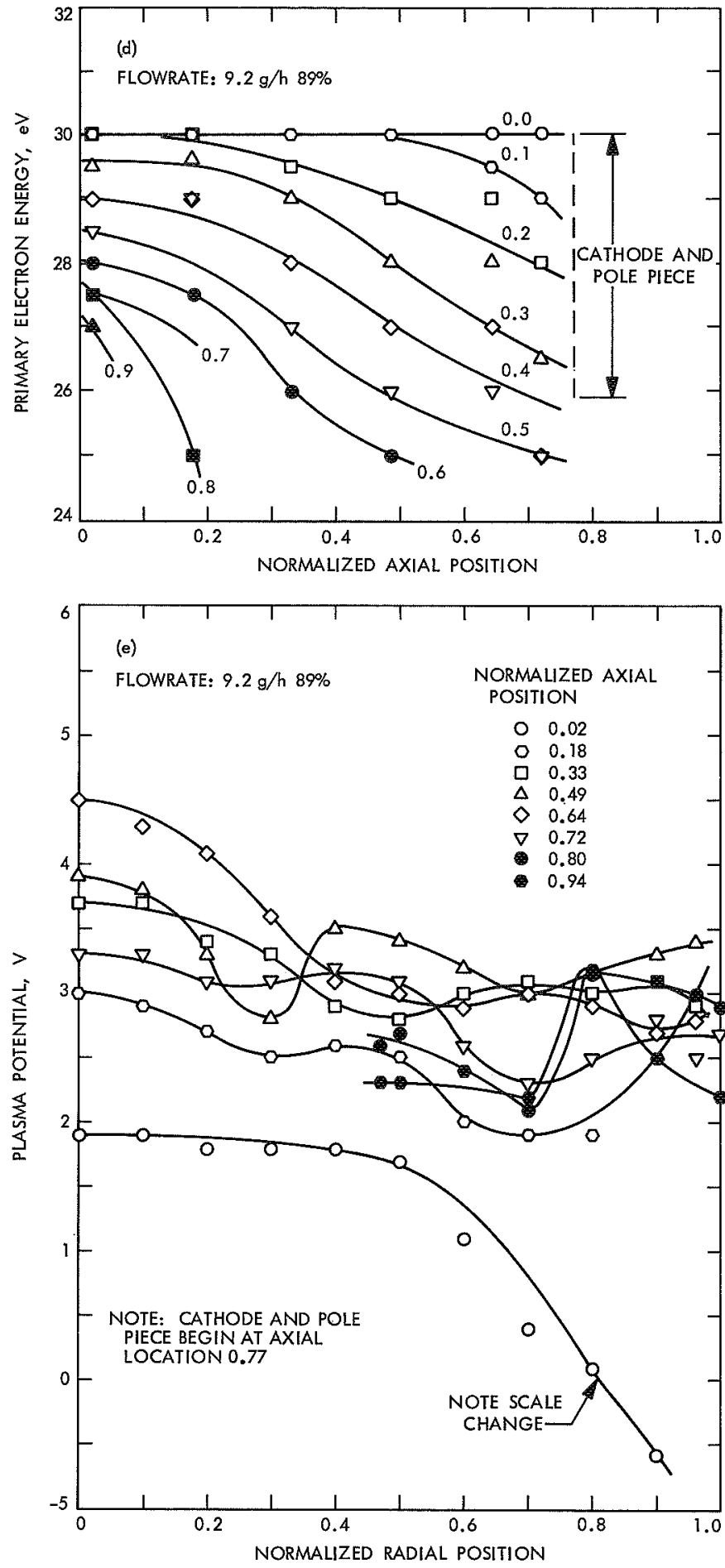

Fig. 7. Plasma properties in the 20-cm-diameter thruster-flowrate, $9.2 \mathrm{~g} / \mathrm{h}$; propellant utilization efficiency, $89 \%$ 


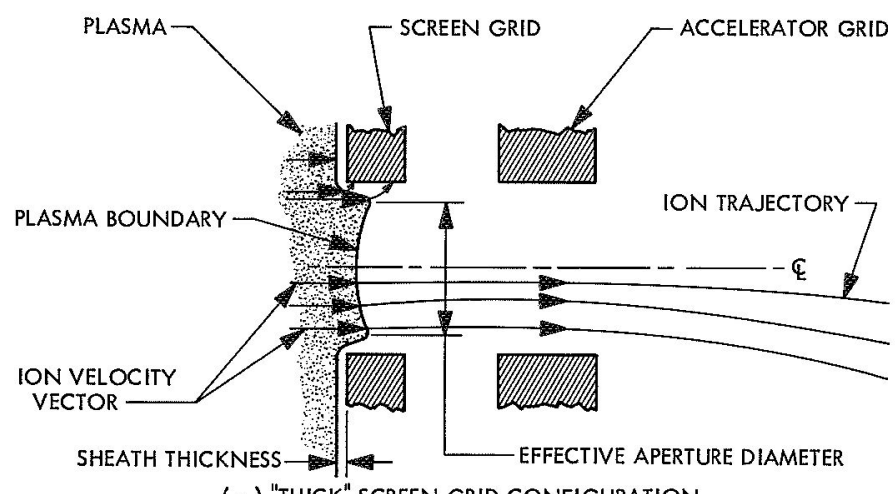

(a) "THICK" SCREEN GRID CONFIGURATION

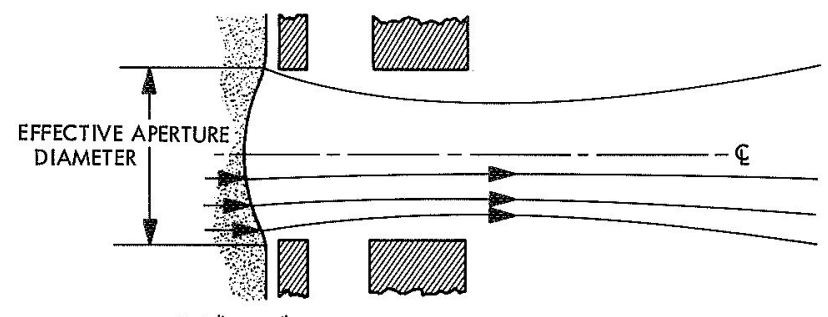

(b) "THIN" SCREEN GRID CONFIGURATION

Fig. 8. Plasma sheath screen grid boundary configurations

The comparison of the measured and calculated beam currents was made by computing the value of $\phi_{i}$ needed to give the correct beam current. The value of $\phi_{i}$ should be approximately equal to the fraction of screen grid open area corrected for sheath effects. The results of calculations using the data in Figs. 2 through 7, as well as four additional sets for the 20 -cm-diameter thruster and two additional, previously published, sets for the $15-\mathrm{cm}$-diameter thruster, are shown in Table 1. Since measurements were not taken at the sheath, the data were extrapolated to this position. The values of $\phi_{i}$ calculated for the $20-\mathrm{cm}$ diameter thruster are quite close to the fraction of screen grid open area of approximately 0.72 (1385 apertures of 0.462 -cm-diameter in a 20 -cm-diameter area). In the 15 -cm-diameter thruster cases, the calculated $\phi_{i}$ value is approximately 0.38 compared with the grid open area fraction of approximately 0.48 ( 475 apertures of $0.475-\mathrm{cm}$ diameter in a 15-cm-diameter area). This difference is directly attributable to the plasma boundary configurations as shown in Fig. 8.

The major accelerator system differences between the $15-\mathrm{cm}$ and $20-\mathrm{cm}$-diameter thrusters studied were the screen grid thickness and the screen-accelerator grid spacing, as indicated in Table 1. The plasma boundary configuration of the $15-\mathrm{cm}$-diameter thruster could correspond to Fig. 8(a), which shows a relatively thick screen with wide spacing. In general, the plasma boundary position adjusts to supply the demanded space-charge-limited current. The space-charge-limited current is inversely proportional to the square of the accelerator-plasma boundary spacing. For a given ion flux density from the plasma, the accelerator-plasma boundary spacing will decrease with decreasing space-charge-limited current demand. This causes the boundary to move toward the accelerator until the total ion arrival at the boundary equals the spacecharge-limited current. For the 15-cm-diameter thruster, this boundary position appears to be within the aperture. Boundary shapes similar to those in Fig. 8 have been found in computer studies of ion optics at Hughes Aircraft (Ref. 15).

With the plasma boundary located within the aperture (Fig. 8a), the effective diameter of the aperture is reduced by approximately twice the plasma-screen grid sheath thickness. Most ions crossing the boundary between the edge of the aperture and the boundary inflection point will be accelerated into the screen grid. The sheath thickness can be estimated by equating the space-chargelimited ion current arriving at the screen to the ion flux from the plasma. Figure 8 defines the terms used for this purpose. For a planar geometry

$$
\frac{4 \epsilon_{0}}{9}\left(\frac{2 e}{m_{i}}\right)^{1 / 2} \frac{V_{s}^{3 / 2}}{x^{2}}=e n_{i} v_{i}
$$

where $V$ is the plasma potential with respect to the screen, $x$ the sheath thickness, and $\epsilon_{0}=8.85 \times 10^{-12} \mathrm{coul} / \mathrm{nm}^{2}$. Solving for $x$, obtaining $v_{i}$ from Eq. (5)

$$
x=\left[\frac{4}{9} \frac{\epsilon_{0}}{e}\left(\frac{2 e}{k T_{e}}\right)^{1 / 2}\left(\frac{n_{m}}{n_{i}}\right)^{1 / 2} \frac{V_{s}^{3 / 2}}{n_{i}}\right]^{1 / 2}, m
$$

Using average plasma conditions at the sheath $(V=35 \mathrm{~V}$, $k T_{e} / e=4 \mathrm{eV}, n_{i}=10^{11} \mathrm{~cm}^{-3}$, and $\left.n_{m} / n_{i}=0.95\right)$ and consistent units, Eq. (8) gives a sheath thickness of approximately $2 \times 10^{-2} \mathrm{~cm}$. The effective aperture diameter in this case (for the 15-cm-diameter thruster) is approximately $0.435 \mathrm{~cm}$, resulting in an effective open area fraction of 0.40 , which is in good agreement with the values calculated from the Bohm ion flux.

A thin-screen grid and close-grid spacing should produce a plasma boundary similar to that shown in Fig. 8(b). It should be noted that in this case $\phi_{i}$ could be greater than the grid open area fraction. This interpretation of the plasma boundary shape change with screen grid thickness is also consistent with efficiency improvements previously observed in tests on the 20-cm-diameter thruster (Ref. 7). 
Table 1. Comparison of ion flux from Bohm criteria with beam current

\begin{tabular}{|c|c|c|c|c|c|c|c|c|c|c|c|}
\hline \multirow{2}{*}{ Paramefer } & \multicolumn{4}{|c|}{ 15-cm-diameter Thrusier } & \multicolumn{7}{|c|}{ 20-cm-diameter Thruster } \\
\hline & 1 & 2 & 3 & 4 & 1 & 2 & 3 & 4 & 5 & 6 & 7 \\
\hline Beam current, A & 0.50 & 0.55 & 0.21 & 0.23 & 0.68 & 0.83 & 1.06 & 1.09 & 0.72 & 0.85 & 0.96 \\
\hline Bohm ion flux, A & 1.35 & 1.44 & 0.54 & 0.62 & 0.90 & 1.10 & 1.41 & 1.48 & 0.95 & 1.15 & 1.30 \\
\hline$\phi_{i}$ & 0.37 & 0.38 & 0.39 & 0.37 & 0.75 & 0.75 & 0.75 & 0.74 & 0.76 & 0.74 & 0.74 \\
\hline \multicolumn{12}{|l|}{ Screen grid open area } \\
\hline fraction & 0.48 & 0.48 & 0.48 & 0.48 & 0.72 & 0.72 & 0.72 & 0.72 & 0.72 & 0.72 & 0.72 \\
\hline$V^{+}, k V$ & 2.5 & 2.5 & 3.5 & 3.5 & 2.0 & 2.0 & 2.0 & 2.0 & 2.0 & 2.0 & 2.0 \\
\hline$V^{-}, k V$ & 2.5 & 2.5 & 2.0 & 2.0 & 2.0 & 2.0 & 2.0 & 2.0 & 2.0 & 2.0 & 2.0 \\
\hline Impingement, mA & 7.5 & 8.0 & 1.5 & 1.5 & 4.3 & 5.6 & 9.4 & 9.6 & 12.8 & 11.9 & 10.6 \\
\hline Discharge current, A & 12.0 & 14.0 & 3.0 & 3.1 & 3.5 & 4.4 & 6.0 & 6.0 & 5.1 & 4.2 & 3.4 \\
\hline Discharge voltage, $\mathrm{V}$ & 33.0 & 35.0 & 45.0 & 4.5 & 34.9 & 35.0 & 34.5 & 35.0 & 35.2 & 35.3 & 35.3 \\
\hline Magnet current, A & 9.0 & 9.0 & 7.5 & 7.5 & 0.7 & 0.7 & 0.7 & 0.7 & 0.7 & 0.7 & 0.7 \\
\hline Propellant flowrate, $\mathrm{g} / \mathrm{h}$ & 4.7 & 4.7 & 1.9 & 1.9 & 5.7 & 6.9 & 9.0 & 9.2 & 9.2 & 9.2 & 9.2 \\
\hline Utilization efficiency, $\%$ & 77.0 & 88.0 & 85.0 & 91.0 & 89.0 & 90.0 & 88.0 & 89.0 & 58.0 & 68.0 & 78.0 \\
\hline Screen thickness, cm & 0.15 & 0.15 & 0.15 & 0.15 & 0.076 & 0.076 & 0.076 & 0.076 & 0.076 & 0.076 & 0.076 \\
\hline Grid spacing, $\mathrm{cm}$ & 0.20 & 0.20 & 0.25 & 0.25 & 0.18 & 0.18 & 0.18 & 0.18 & 0.18 & 0.18 & 0.18 \\
\hline Anode diamefer, cm & 14.5 & 14.5 & 14.5 & 14.5 & 20.3 & 20.3 & 20.3 & 20.3 & 20.3 & 20.3 & 20.3 \\
\hline Chamber length, $\mathrm{cm}$ & 9.8 & 9.8 & 10.7 & 10.7 & 16.3 & 16.3 & 16.3 & 16.3 & 16.3 & 16.3 & 16.3 \\
\hline Cathode type & $\mathbf{a}$ & $\mathbf{a}$ & $\mathbf{b}$ & b & c & c & $c$ & $c$ & $c$ & $c$ & c \\
\hline
\end{tabular}

From the agreement of the calculated value of $\phi_{i}$ with the corrected grid open area fraction, it can be concluded that, within the experimental accuracy of the present data, the ion velocity at the sheath is given by the modified Bohm criterion. Ion fluxes to the anode, cathode, and housing can now be computed using this ion velocity and the plasma conditions at the location of interest.

2. Wall flux. The factor $\omega$ in Eq. (2) is the ratio of the total ion flux (to all boundaries including the beam) to the beam current. The ion current to any surface in the thruster can be calculated by integrating the flux $\left(e n_{i} v_{i}\right)$ over that surface. As before, this integration was accomplished by summing the flux contributions to segments of area. The anode, housing, rear chamber surface, cathode, and cathode pole piece surfaces were divided into segments. The size of the segment was chosen to be consistent with the variation of $\left(n_{i} v_{i}\right)$ along that segment. The values of $n_{i}$ and $v_{i}$ (or $k T_{e} / e$ ) at the surface (i.e., the sheath at the surface) were found, as before, by extrapolating the experimental data graphically to the surface and using the Bohm criterion.

The results of these calculations, using the data of Figs. 2 through 7 as well as one additional data set for the 20-cm-diameter thruster and one additional set for the 15-cm-diameter thruster, are shown in Table 2 . The values of $\omega$ thus computed allow $\epsilon_{b}$ to be found since $\epsilon_{t}$ is known. These values of $\epsilon_{b}$ are also given in Table 2 . A comparison of the $15-\mathrm{cm}$ and $20-\mathrm{cm}$-diameter thruster results, and a discussion of the relationship of these to performance, will be presented later in this report.

\section{Electron Flux}

The total flux of electrons to the walls must balance the total ion flux for all surfaces but the anode. The net current to the housing (cathode, pole piece, rear surface, screen grid, etc.) must be zero. Total electron current to the anode is the sum of the discharge, beam, and ion currents. Since the discharge and beam currents are directly measured, a calculation of the electron current to the anode should verify the previous ion flux calculation.

The anode sheath retards electron flow and is similar to that of a negatively biased Langmuir probe. Thus, to first order, the electron flux to the anode is given by

$$
\Gamma_{e}=\frac{\left(\boldsymbol{n}_{e} \bar{v}_{e}\right)_{s}}{4} e^{-\alpha_{1}^{2}}
$$


Table 2. Summary of ion wall-flux calculations

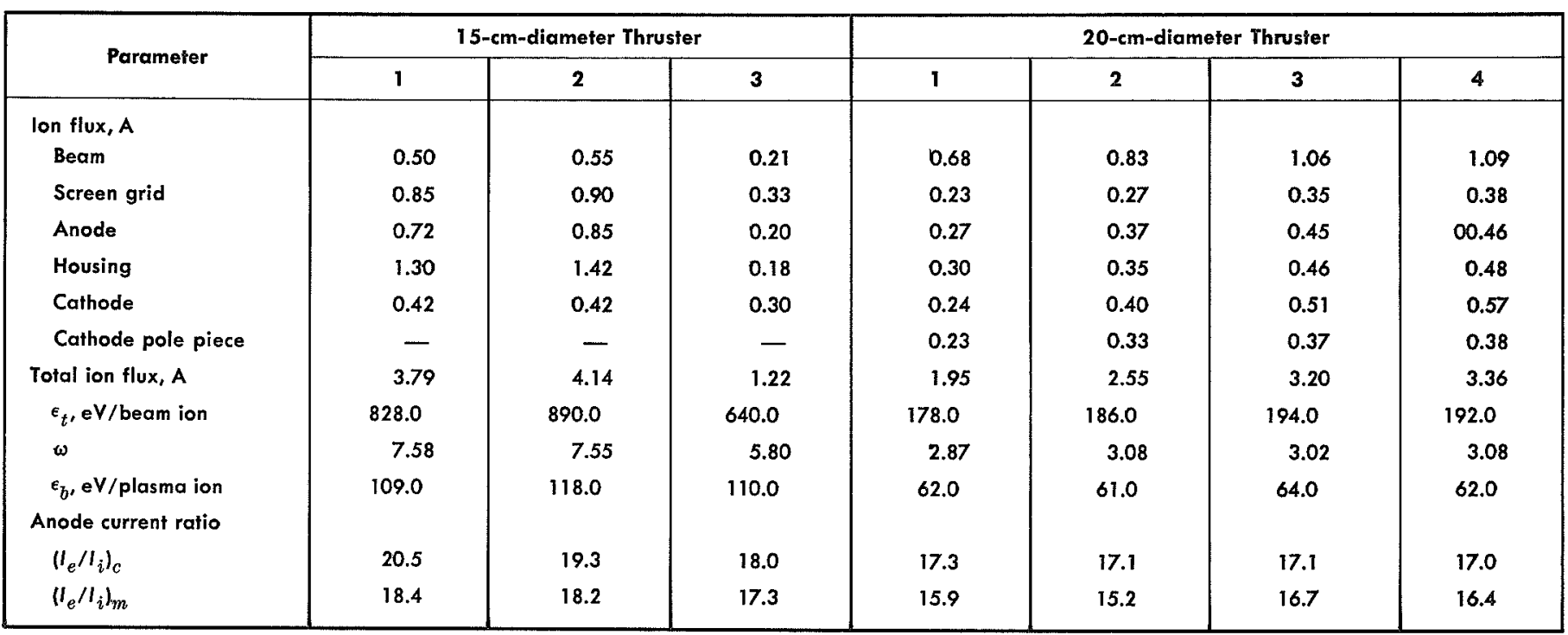

where

$$
\begin{aligned}
& \alpha_{1}=\frac{\left(v_{s}\right)_{n}}{c_{e}} \\
& c_{e}=\left(\frac{2 k T_{e}}{m_{e}}\right)^{1 / 2}=\left(\frac{\pi}{2}\right)^{1 / 2} \bar{v}_{e}
\end{aligned}
$$

and $\left(v_{s}\right)_{n}$ is the minimum electron velocity, in a direction normal to the sheath, needed to overcome the sheath potential. The ratio of electron to ion fluxes is from Eqs. (5) and (9)

$$
\frac{\Gamma_{e}}{\Gamma_{i}}=244 e^{-\alpha_{1}^{2}}
$$

Since density and temperature drop out of Eq. (10), and $\Gamma_{i}$ is already known, finding the value of this ratio is equivalent to finding $\Gamma_{e}$.

The flux ratio is not necessarily constant along the anode. Thus, the flux ratio at a given location probably does not equal the total current ratio $\left(I_{e} / I_{i}\right)$. Equation (10) was evaluated for each anode area segment as in the ion flux calculations. These values were averaged by area and summed to obtain the average total current ratio. These calculated values $\left(I_{e} / I_{i}\right)_{c}$ are presented in Table 2. "Measured" values $\left(I_{e} / I_{i}\right)_{m}$ of the ratio were found by taking the ratio of total electron current (discharge, beam, and ion currents) to calculated ion current. The agreement between these values is well within the experimental accuracy and shows that the calculations of electron and ion flux are consistent.

\section{Ion Production Rate}

The total ion production rate within the plasma must equal the total ion flux. Thus, a direct calculation of the ion production rate is a second method for evaluating $\omega$ and $\epsilon_{b}$.

With the assumption that ionization occurs only from the ground state, the ion production rate can be found from previous calculations by Kerrisk (Ref. 16). The ion production rate can be written in the form (Ref. 2)

$$
\dot{\nu}_{i}=n_{0}\left[n_{m} \Sigma_{m}+n_{p} \Sigma_{p}\right], \frac{\text { ions }}{\mathrm{s} \mathrm{cm}^{3}}
$$

where $\Sigma_{m}$ and $\Sigma_{p}$ are coefficients for Maxwellian and primary electrons, respectively, and $n_{0}$ is the neutral atom density. It should be noted that the densities and coefficients in Eq. (11) are functions of position, and that $\dot{v}_{i}$ must be integrated (or summed) over the plasma volume to find the total ion production rate.

A major difficulty in evaluating $\dot{v}_{i}$ is that $n_{0}$ is unknown. A first-order estimate of this density can be made as discussed below. The neutral flux at any location in the plasma must satisfy the mass conservation equation

$$
\nabla \cdot \vec{\Gamma}_{0}=-\dot{v}_{i}
$$

where $\vec{\Gamma}_{0}$ is the neutral flux vector. The neutral atom mean free path is greater than thruster dimensions except for atom-electron collisions. However, only ionizing collisions 
significantly affect the atom motion. The mean free path of an atom prior to ionization is given by

$$
\lambda_{\mathrm{oe}}^{*}=\frac{\bar{v}_{0}}{\left[n_{m} \Sigma_{m}+n_{p} \Sigma_{p}\right]}
$$

where $\bar{v}_{0}$ is the atom mean thermal speed equal to approximately $2.5 \times 10^{4} \mathrm{~cm} / \mathrm{s}$ for a temperature of $600^{\circ} \mathrm{K}$. Since most atoms originate from chamber surfaces, this is a reasonable temperature. Choosing average plasma conditions $\left(n_{m}=5 \times 10^{11} \mathrm{~cm}^{-3}, k T_{e} / e=4 \mathrm{eV}, n_{p}=10^{10} \mathrm{~cm}^{-3}\right.$, and $\left.\epsilon_{p}=30 \mathrm{eV}\right)$, the coefficients are $\Sigma_{m}=6 \times 10^{-9}$ and $\Sigma_{p}=$ $1.6 \times 10^{-7} \mathrm{~cm}^{3} / \mathrm{s}$, giving $\lambda_{0 e}^{*}=5.5 \mathrm{~cm}$. Since the densities and temperatures decrease near the walls, $\lambda_{0 e}^{*}$ is longer in these regions. However, few atoms can pass directly through the plasma central region without being ionized.

Since most atoms originate at the walls and evaporate randomly, it will be assumed for this analysis that the radial and axial atom flux components are given by

$$
\left.\begin{array}{l}
\Gamma_{0}^{r}=-\frac{1}{4} n_{0}(r, z) \bar{v}_{0} \\
\Gamma_{0}^{z}=\frac{1}{4} n_{0}(r, z) \bar{v}_{0}
\end{array}\right\}
$$

These correspond to random fluxes off the walls and should be reasonably correct near the walls. The geometry is illustrated in Fig. 9. The negative sign occurs in the radial flux component because the radial direction is taken as positive outward. The axial flux is taken to be positive since $z=0$ is the chamber rear surface and a net atom flux toward the grid exists. Azimuthal symmetry is assumed.

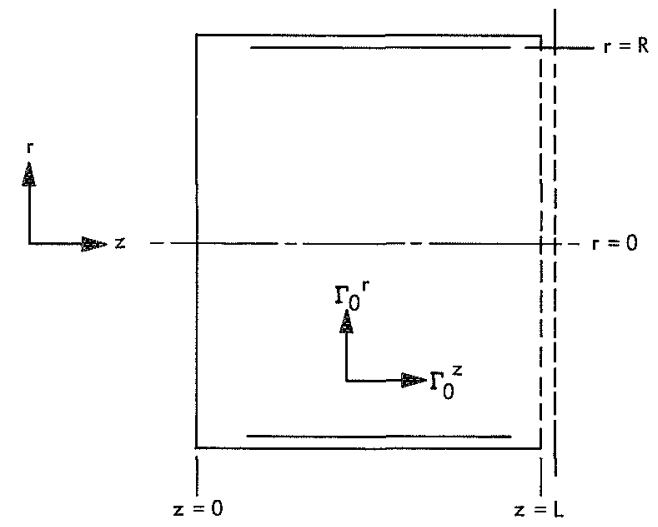

Fig. 9. Configuration for neutral atom density analysis
Since Eq. (14) is valid only near the walls, the solution of Eq. (12) is divided into two regions: center region $\left(r<r_{0}\right)$ and wall region $\left(r_{0}<r<R\right)$. The value of $r_{0}$ is arbitrarily taken to be $R / 2$. The radial density distribution to the center region is assumed to be constant. The effect of these assumptions will be assessed later.

Substituting Eqs. (14) into Eq. (12), and noting that $\dot{v}_{i}$ is given by Eq. (11), gives

$$
\frac{1}{r} \frac{\partial}{\partial r}\left(r n_{0}\right)-\frac{\partial n_{0}}{\partial z}=\frac{4 n_{0}}{\lambda_{0 e}^{*}}
$$

This will be solved by assuming

$$
n_{0}=R_{0}(r) Z_{0}(z)
$$

using the boundary conditions

$$
\begin{gathered}
\frac{\partial n_{0}}{\partial r}=0 \quad \text { at } \quad r=r_{0} \\
\frac{\pi}{4} \bar{v}_{0} m_{0} \phi_{0}\left[2 \int_{r_{0}}^{R} n_{0}(r, L) r d r+r_{0}^{2} n_{0}\left(r_{0}, L\right)\right]= \\
\left(1-\eta_{m}\right) \dot{m} \quad \text { at } \quad z=L
\end{gathered}
$$

where $r_{0}$ is the boundary between the center and wall solution regions, $R$ the chamber radius, $\eta_{m}$ the thruster propellant utilization efficiency, $\dot{m}$ the propellant flowrate, and $\phi_{0}$ the fraction of grid open area for atom flow. An average mean free path $\lambda_{0 e}^{*}=\lambda$ will be used since the solution is quite approximate.

The solution of Eq. (15) can be written as

$$
n_{0}(r, z)=n_{0}\left(r_{0}, L\right) \frac{r_{0}}{r} \frac{e^{r /\left(r_{0}\right)-\beta z}}{e^{1-\beta L}}
$$

where $n_{0}\left(r_{0}, L\right)$ is the atom density at the screen grid for $r<r_{0}$ and is given by

$$
n_{0}\left(r_{0}, L\right)=\frac{3.05 \times 10^{17}\left(1-\eta_{m}\right) \dot{m}}{r_{0}^{2}\left(2 e^{R-r_{0} / r_{0}}-1\right)}
$$

and

$$
\beta=\frac{4}{\lambda}-\frac{1}{r_{0}}=\frac{4}{\lambda}-\frac{2}{R}
$$


Since few atoms can travel a distance greater than $R$ (at least radially), an average mean free path $\lambda=R$ appears to be a reasonable choice for the present data. With these values for $\lambda$ and $r_{0}, \beta$ is approximately 0.2 . The ratio of $n_{0}(r, z) / n_{0}\left(r_{0}, L\right)$ is shown in Fig. 10.

Applying Eqs. (11), (16), and (17) to the data sets used in the previous section, the local ion production rates were computed. The local rates were multiplied by volume elements and summed to find the total rate. The values of $\omega$ and $\epsilon_{b}$ so determined are presented in Table 3. The reasonable agreement between the results in Table 2 and Table 3 gives increased confidence in both methods. However, the crude atom density calculation places an upper limit on the confidence in the ion production method. Probably the most significant result is that the calculated atom density must be of the correct order of magnitude. This result justifies the assumptions for $r_{0}$ and $\lambda$. Since the

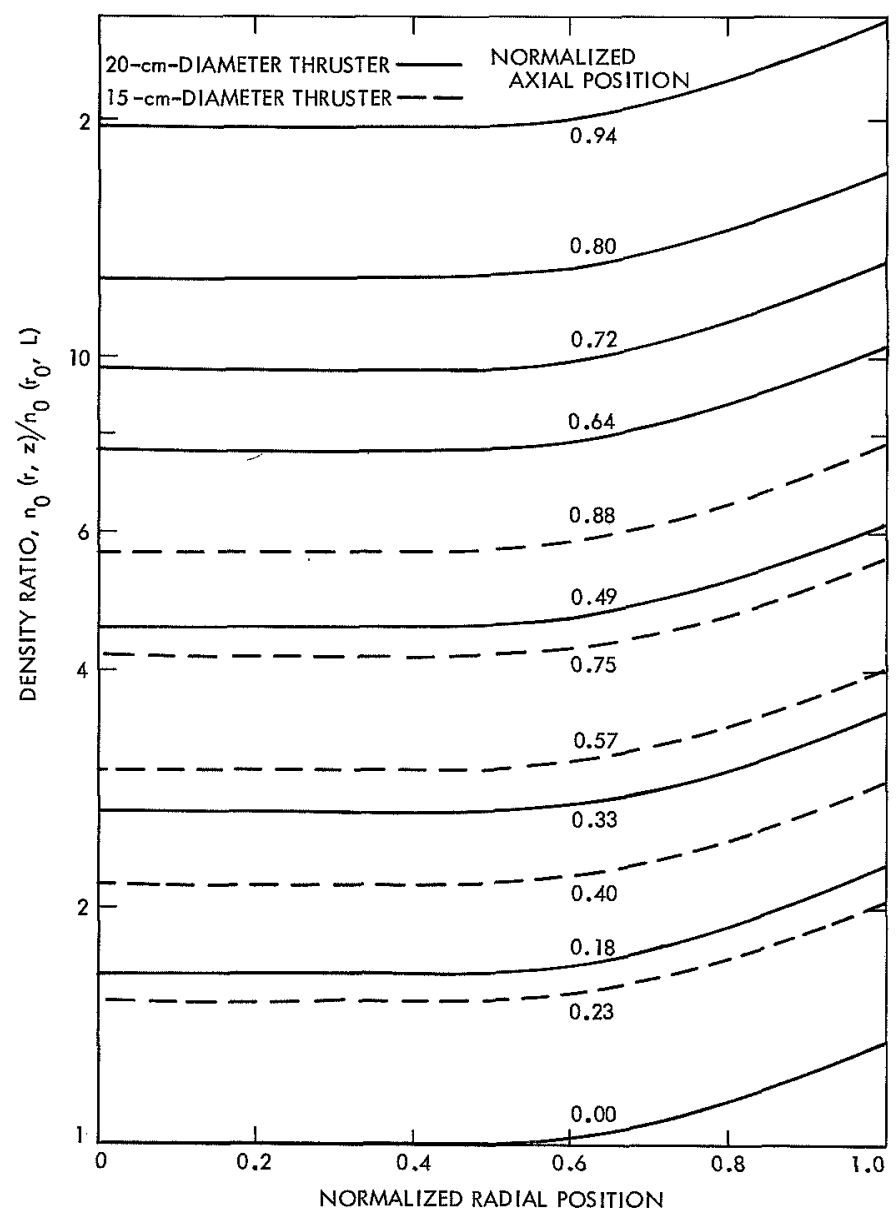

Fig. 10. Ratio of atom density at a given position to that at the center grid position
Table 3. Summary of ion production rafe calculations

\begin{tabular}{|l|r|c|c|c|c|c|}
\hline \multirow{2}{*}{ Parameter } & \multicolumn{2}{|c|}{$\begin{array}{r}\text { 15-cm-diameter } \\
\text { Thruster }\end{array}$} & \multicolumn{4}{|c|}{20 -cm-diameter Thrusier } \\
\cline { 2 - 7 } & \multicolumn{1}{|c|}{1} & \multicolumn{1}{c|}{2} & 1 & 2 & 3 & 4 \\
\hline Beam current, A & 0.50 & 0.55 & 0.68 & 0.83 & 1.06 & 1.09 \\
$\phi_{0}$ & 0.5 & 0.5 & 0.44 & 0.44 & 0.44 & 0.44 \\
$n_{0}\left(r_{0}, l\right) \times 10^{-11}, \mathrm{~cm}^{-3}$ & 12.0 & 7.6 & 4.8 & 5.2 & 8.0 & 7.0 \\
Total ion production & & & & & & \\
rate, A & 4.5 & 4.5 & 1.8 & 2.8 & 2.9 & 3.6 \\
$\omega$ & 9.0 & 8.2 & 2.7 & 3.4 & 2.7 & 3.3 \\
$\epsilon_{t}$ & 828.0 & 890.0 & 178.0 & 186.0 & 194.0 & 192.0 \\
$\epsilon_{b}$ & 92.0 & 109.0 & 66.0 & 55.0 & 69.0 & 58.0 \\
\hline
\end{tabular}

total production rate was found by summing average rates, the actual atom distribution could be somewhat different from that used. An accurate measurement of the atom density would be useful, however, such a measurement seems difficult.

Having confirmed the general accuracy of the total ion production rate, the distribution of local rates should be noted. These rates, from both Maxwellian and primary electrons, are shown in Fig. 11 for data from the $20-\mathrm{cm}$ diameter thruster. The ionization rate due to primary electrons is shown in Fig. 12. The figure illustrates that primary electrons contribute substantially to the total ion production.

\section{E. Ion Production Cost}

The basic mechanisms for energy loss from the plasma can be discussed by considering the energy lost by electrons. This is possible because the discharge power is initially totally contained in the electron energy. Electrons lose energy mainly in inelastic collisions with atoms and ions and in collisions with chamber surfaces. Elastic collision losses are quite small. The sum of the inelastic collisional loss and the surface loss will be compared with the total discharge loss.

The chamber (anode and other housing potential surfaces) losses can be estimated as follows. The average electron energy transported across the sheath can be found by integrating over the electron velocity distribution function for electron energies greater than the sheath potential $V_{s}$ near the walls, the electron velocity distribution function is generally Maxwellian (see Figs. 2 through 7). The 


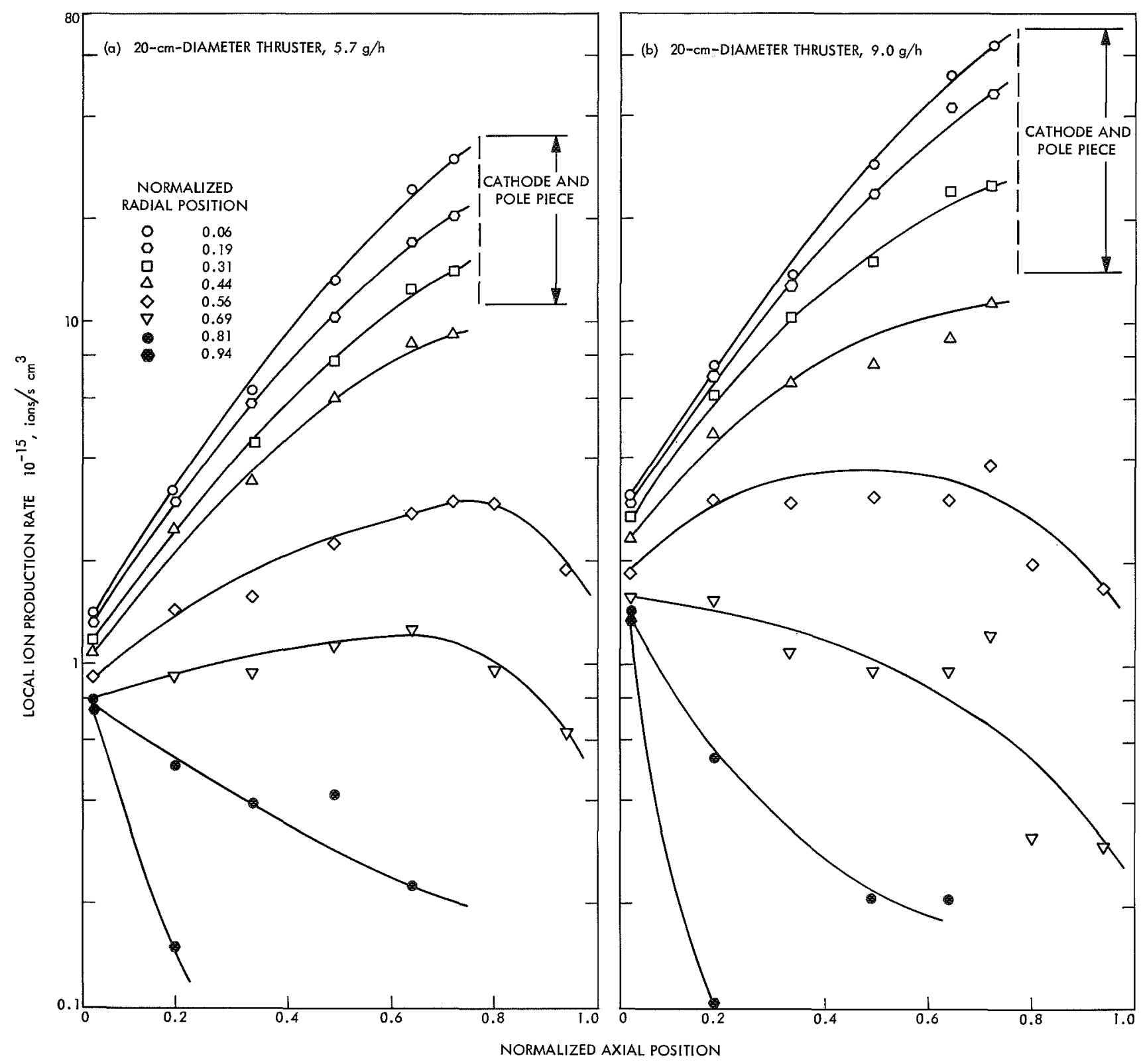

Fig. 11. Local ion production rate by Maxwellian and primary electrons 


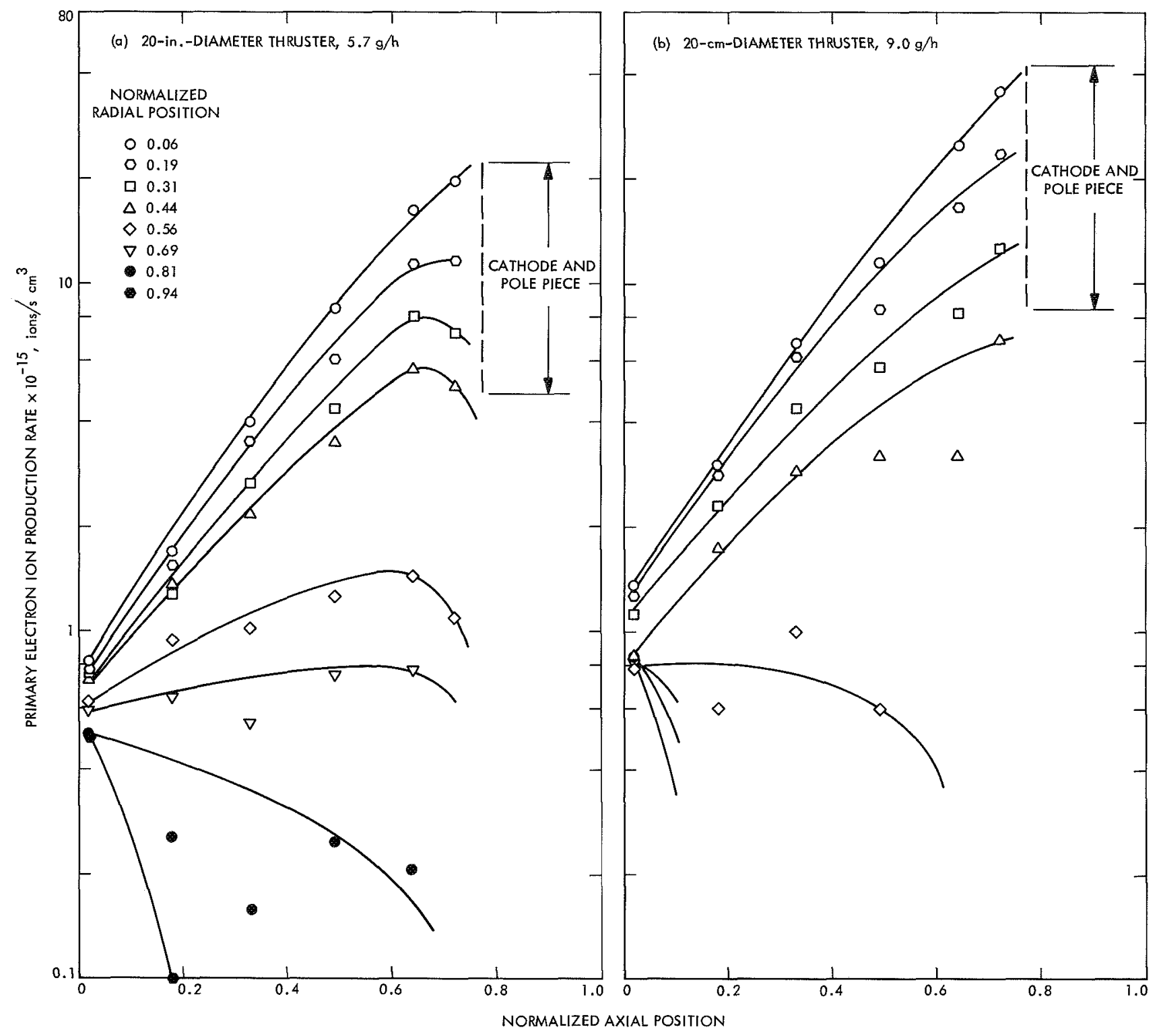

Fig. 12. Local ion production rate by primary electrons

average electron energy flux reaching the wall is given by

$$
q_{e}=\frac{2 k T_{e}}{e} e \Gamma_{e} G, W
$$

where

$$
G=1-\frac{(\pi)^{1 / 2}}{2} \alpha_{1}\left(1-\operatorname{erf}\left(\alpha_{1}\right)\right) e^{\alpha_{1}^{2}}
$$

and other terms were defined for Eq. (9). The factor G does not vary greatly from unity and, in the present analy- sis, will be taken equal to unity. It should be noted that $e \Gamma_{e}$ is just the electron current to the wall.

The chamber losses can be computed from Eq. (18) using the previously tabulated wall fluxes. These losses are given in Table 4 for the anode and housing surfaces. The housing loss neglects the effect of primary electrons. Average Maxwellian electron temperatures near the walls were used for the calculations.

The average energy loss due to inelastic electron collisions $\epsilon_{c}(\mathrm{eV} /$ plasma ion) can be estimated by considering the energy lost in ionization and excitation of atoms and 
ions. For the present density range, most of the energy going into excitation should be radiated to the walls (Ref. 17). The local collisional loss, $\epsilon_{c}^{\prime}$, which will be a function of position in the plasma, is written in the form (Ref. 17)

$\epsilon_{c}^{\prime}=V_{i}+\frac{\sum_{j}<Q_{j}\left(\epsilon_{e}\right) \epsilon_{e}^{1 / 2}>V_{j}+\frac{n_{i}}{n_{0}} \sum_{k}<Q_{k}\left(\epsilon_{e}\right) \epsilon_{e}^{1 / 2}>V_{k}}{\left\langle Q_{i}\left(\epsilon_{e}\right) \epsilon_{e}^{1 / 2}>\right.}$

where $V_{i}$ is the ionization potential, $V_{j}$ the energy of the $j$ th atomic state, $V_{k}$ the energy of the $k$ th ion state, $Q_{i}$ the ionization cross section, $Q_{j}$ the atom excitation cross section for state $j, Q_{k}$ the ion excitation cross section for state $k, \epsilon_{e}$ is the electron energy, and the brackets indicate an integration over the energy distribution function. Thus, in the right-hand term of Eq. (19), the numerator represents the excitation loss and the denominator represents the ionization rate.

The excitation loss in Eq. (19) was evaluated for Maxwellian and primary electron energy distributions and $\epsilon_{c}^{\prime}$ is shown in Fig. 13 for these distributions. The atom excitation terms were evaluated for the $6{ }^{1} P_{1}$ and ${ }^{3} P_{1}$ states which have transitions to the ground state. The cross sections for these distributions were taken from Ref. 18. Other atomic terms should be small. Ion excitation cross sections were not available; however, the ion contribution can be estimated. Line intensities for ion transitions (Ref. 19) are small except for the $1942 \AA$ and $2345 \AA$ lines. The intensity of these lines is about the same as for the ${ }^{1} P_{1}(1849 \AA)$ atom line.

As an estimate of the ion excitation contribution to $\epsilon_{c}^{\prime}$, the ion excitation loss was taken to be equal to that for the ${ }^{1} P_{1}$ atom state. A value of $n_{i} / n_{0}$ equal to unity was used in computing the curves of Fig. 13. The curves could be expected to shift upward or downward as $n_{i} / n_{0}$ is increased or decreased. The ion production rates were computed from the $\Sigma_{m}$ and $\Sigma_{p}$ coefficients. The results are consistent with similar calculations of Dugan and Sovie (Ref. 17) for cesium, argon, and helium. The mercury $\left(V_{i}=10.39 \mathrm{eV}\right)$ curves lie between those for cesium $\left(V_{i}=3.87 \mathrm{eV}\right)$ and $\operatorname{argon}\left(V_{i}=15.76 \mathrm{eV}\right)$.

The average loss $\epsilon_{c}$ cannot be evaluated directly because the Maxwellian electron temperature and primary electron energy distribution vary spacially (i.e., $\epsilon_{c}^{\prime}$ varies spacially). The contributions to ionization at a given posi-

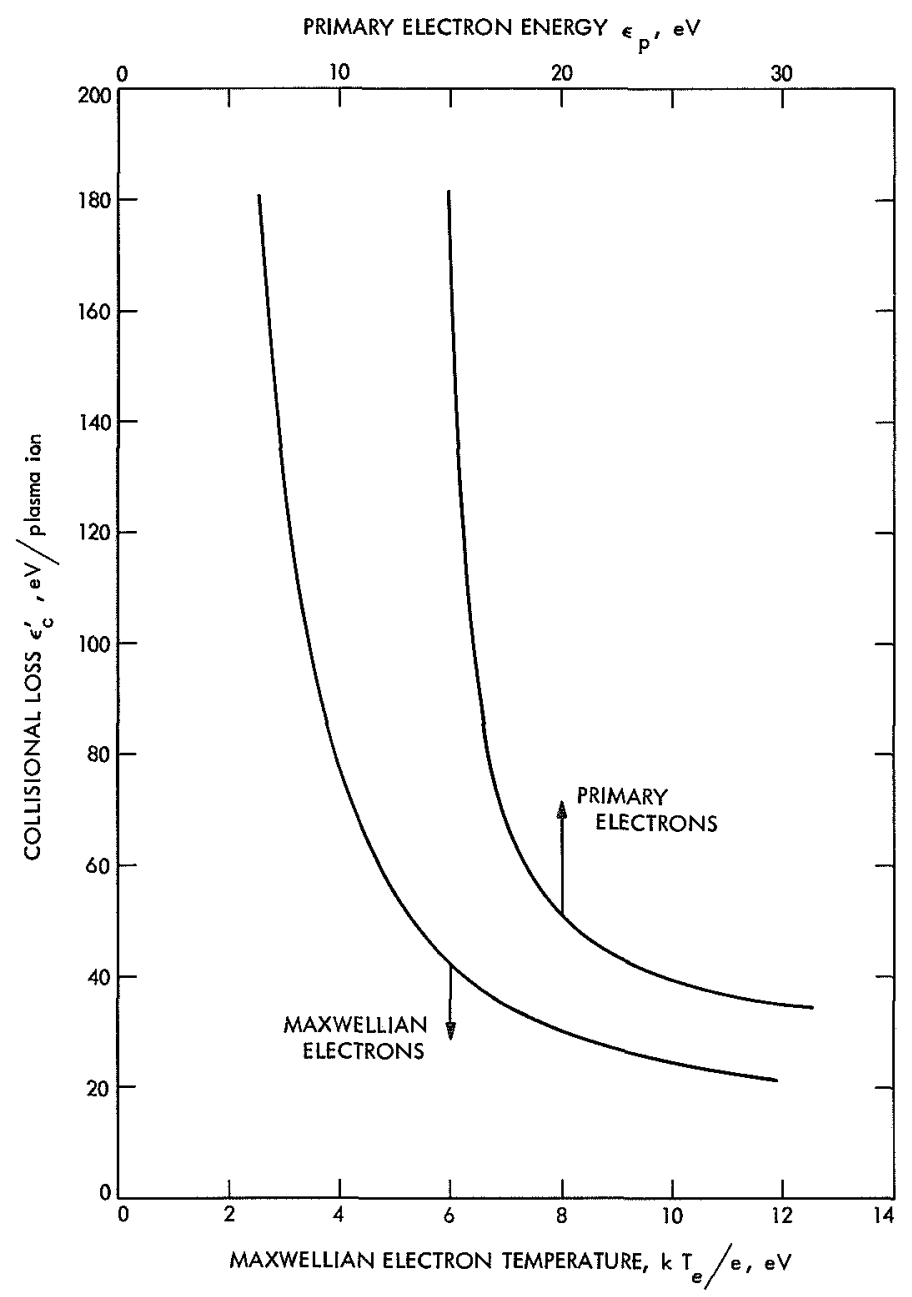

Fig. 13. Local collisional energy loss factor

tion must be weighted by volume. The discharge power should equal the integrated product over the chamber of $\epsilon_{c}^{\prime} \dot{v}_{i}$ plus the anode and housing loss. Using the data presented previously in preparing Table $3, \epsilon_{c}^{\prime} \dot{v}_{i}$ was summed over the chamber volume elements to obtain the results shown in Table 4. This calculation of the discharge power resulted in values within $20 \%$ of the measured power. This is a reasonable variation considering that the $\epsilon_{c}^{\prime}$ curves used (Fig. 13) do not account for changes in $n_{i} / n_{0}$.

\section{F. Discussion}

The previous analysis provided relationships for relating the plasma properties to thruster performance. The fundamental importance of the discharge loss factors $\omega$ and $\epsilon_{b}$ was demonstrated. The validity of the Bohm criterion for calculating wall ion fluxes and the wall loss factor $\omega$ were verified by computing (1) the ion beam current, 
Table 4. Electron energy losses

\begin{tabular}{|l|r|r|r|r|r|r|}
\hline \multirow{2}{*}{ Parameter } & \multicolumn{2}{|c|}{$\begin{array}{r}\text { 15-cm-diameter } \\
\text { Thruster }\end{array}$} & \multicolumn{3}{|c|}{20 -cm-diameler Thruster } \\
\cline { 2 - 7 } & \multicolumn{1}{|c|}{1} & \multicolumn{1}{c|}{2} & \multicolumn{1}{c|}{1} & \multicolumn{1}{c|}{2} & \multicolumn{1}{c|}{4} \\
\hline Anode loss, W & 53 & 62 & 8 & 10 & 11 & 17 \\
Housing loss, W & 17 & 20 & 5 & 6 & 6 & 6 \\
Collisional loss, W & 290 & 350 & 120 & 166 & 210 & 214 \\
Discharge power, W & 413 & 490 & 122 & 154 & 206 & 209 \\
$\epsilon_{a^{\prime}}$ eV/plasma ion & 14 & 15 & 4 & 4 & 3 & 5 \\
$\epsilon_{h^{\prime}}$ eV/plasma ion & 5 & 5 & 2 & 2 & 2 & 2 \\
$\epsilon_{c^{\prime}}$ eV/plasma ion & 76 & 85 & 60 & 65 & 66 & 66 \\
"Theorefical" $\epsilon_{b}$ & 95 & 105 & 66 & 71 & 71 & 73 \\
\hline
\end{tabular}

(2) the total ion production rate, and (3) the anode current. The basic cost of ion production, $\epsilon_{b}$, was shown to be the sum of electron inelastic collisional (radiation and ionization) losses and electron energy transport to the walls.

Improvements in thruster performance must involve the factors $\omega$ and $\epsilon_{b}$. This is illustrated in Table 2. Considering the Bohm criterion, $\omega$ is reduced by (1) increasing the ratio of effective screen grid open area to wall area, (2) selectively increasing the electron temperature in the grid region or decreasing this temperature at the walls, and (3) selectively increasing the ion density in the grid region or reducing this density at the walls. The basic ion production cost depends on electron energy flux losses and collisional losses. The electron energy flux loss is reduced by (1) reducing the wall flux, and (2) reducing the electron temperature at the wall. The collisional loss is reduced by (1) decreasing the ion density (since $\epsilon_{c}^{\prime} \dot{v}_{i}$ is proportional to ion density), and (2) increasing the electron energy throughout the plasma. It is noted that selective reductions in ion density can decrease both $\omega$ and $\epsilon_{b}$. However, a reduction in electron energy to reduce the wall flux will result in a large increase in $\epsilon_{c}^{\prime}$. This indicates that the lower limit on discharge losses will be determined primarily by the tradeoff of wall losses and basic ion production costs.

\section{Effects of Thruster Configuration}

The previous analysis can be applied to provide an explanation of recent improvements in thruster discharge performance (Refs. 6, 7). These improvements resulted from configuration changes, and, hence, from changes in the plasma characteristics. The discussions that follow assess these past improvements and possibilities for future improvement.

The major configuration factors affecting thruster performance are magnetic field shaping elements (cathode and screen grid pole pieces), cathode position, screen grid (thickness and open area), and propellant introduction method. The observed effect of changes in these factors, in terms of discharge loss change, will be compared with expected loss changes. Other configuration changes can be evaluated by comparison with these major factors.

The studies of Refs. 6 and 7 were conducted by making modifications to a given thruster. To compare the improvements with the present analysis, the modifications must be considered in the sequence performed. The plasma conditions change with each modification. Therefore, the plasma conditions used in the analysis to consider a given modification or loss change must consider the previous modifications. The 15-cm-diameter thruster used here was similar in performance and configuration to the "unimproved" or original $20-\mathrm{cm}$-diameter thruster. Therefore, the 15-cm-diameter thruster plasma data will be used as a reference for evaluating the improvements in the $20-\mathrm{cm}$ diameter thruster.

\section{A. Pole Pieces}

Although the magnetic field is shaped by both the ferromagnetic cathode and screen grid pole pieces, shown in Fig. 14, the cathode pole piece is most important (Ref. 7). With the cathode mounted at the rear of the chamber, a cathode pole piece was added. This addition reduced the discharge losses by approximately $100 \mathrm{eV} /$ beam ion. However, it must be noted that this loss change represents only

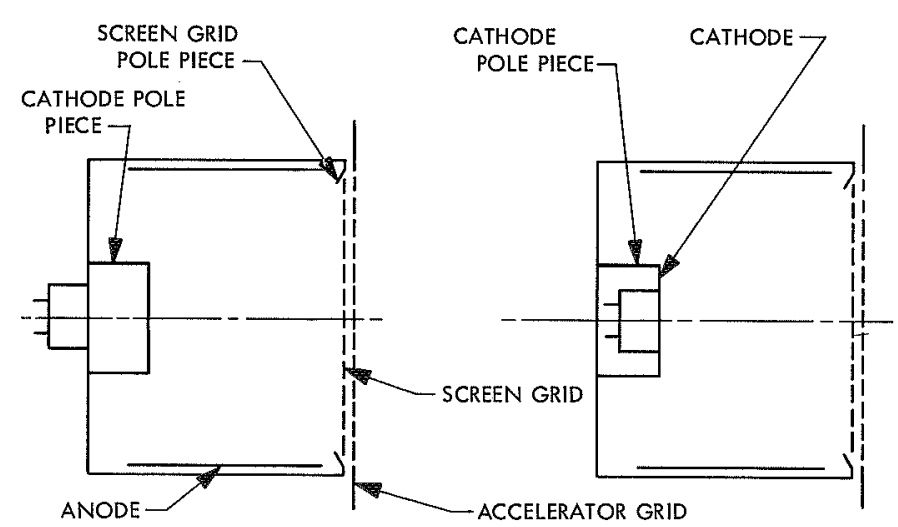

(a) REAR POSITION

(b) FORWARD POSITION

Fig. 14. Cathode and pole piece configurations 
part of the pole piece contribution. A loss is incurred, as discussed in the following section, by placing the cathode at the rear of the pole piece. Had the cathode been mounted at the forward end of the pole piece originally, a loss reduction of approximately $280 \mathrm{eV} /$ ion would have occurred in adding the pole piece. Therefore, this $280 \mathrm{eV} /$ ion change must be explained here. The fact that approximately $180 \mathrm{eV} /$ ion of this improvement can be lost by cathode positioning will be discussed later.

The cathode pole piece increases the axial magnetic field strength in the central region of the discharge. This field localization reduces the radial drift of electrons, particularly primary electrons. A reduced radial electron drift lowers the electron density and temperature in the outer wall and anode region, but maintains the plasma conditions at the grid.

The reduced density and temperature simultaneously reduce the ion production rate and the ion wall flux. In this process, the wall loss factor $\omega$ is reduced. The discharge is then maintained with lower discharge current for the given beam current.

The basic ion production cost $\epsilon_{b}$ is reduced in this process. The local collisional power loss $\epsilon_{c}^{\prime} \dot{v}_{i}$ depends directly on ion density and, therefore, a general decrease in density decreases this loss. The electron energy transport contributions to $\epsilon_{b}$ are also reduced by the reduced anode electron current and by all ion wall currents.

It should be noted from Figs. 2 through 5 and Tables 2 and 4 that ion density, electron temperature, $\omega, \epsilon_{c}, \epsilon_{a}, \epsilon_{h}$, and $\epsilon_{b}$ are all lower in the improved 20-cm-diameter thruster compared with the 15-cm-diameter thruster. Although the initial plasma properties of the unimproved $20-\mathrm{cm}$ diameter thruster may have been slightly different from the 15-cm-diameter thruster, the changes in plasma conditions and loss factors are as expected.

An $\omega$ for the unimproved thruster of approximately 7.5, as for the reference 15-cm-diameter thruster (columns 1 and 2 of Table 2), and an $\epsilon_{b}$ of approximately 100 account for the 700 to $800 \mathrm{eV} /$ beam ion losses observed in Ref. 7 (for 80 to $90 \%$ propellant utilization efficiency). The major changes in $\epsilon_{b}$ and $\omega$ (i.e., $\epsilon_{t}$ ) occurred with the addition of the pole piece and the change in screen thickness. Since plasma measurements are available only for the final 20-cm-diameter thruster, the individual loss factor changes cannot be separated. It will be assumed that the change in $\epsilon_{b}$ for the pole piece addition is half the total change (about $20 \mathrm{eV} /$ plasma ion). This requires an $\omega$ change of 1.5 to 2.0 to obtain the measured losses (i.e., 440 to $480 \mathrm{eV} /$ ion compared with the measured losses of 420 to $520 \mathrm{eV} /$ ion). Considering the expected density change at the walls and the expected reductions in collisional and transport losses, these changes in $\omega$ and $\epsilon_{b}$ seem reasonable.

\section{B. Cathode Position}

After adding a cathode pole piece, the cathode was moved from the rear to the forward end of the pole piece (Ref. 7) (see Fig. 14). A reduction in discharge losses of approximately $180 \mathrm{eV} /$ beam ion was found. In addition, a cathode position near the end of the piece produced the best discharge performance in tests of Ref. 20.

These observations can be accounted for by considering the ion wall losses to the pole piece. With the cathode inside the pole piece, a high-temperature, high-density plasma should exist in the pole piece. As before, the ion wall flux will be that specified by the Bohm criterion. Movement of the cathode to the front of the pole piece eliminates this ion loss and results in reduced discharge losses. The power loss can be estimated by assuming reasonable plasma conditions in the pole piece. Since the flux varies with the square root of $k T_{e} / e$ and $n_{i} / n_{m}$, the choice of these quantities is not too important. However, the choice of the ion density and $\epsilon_{b}$ has a strong influence on the result. Therefore, the value of the quantity $\left(\epsilon_{b} n_{i}\right)$ needed to produce a loss change of $180 \mathrm{eV} /$ ion will be determined. The equation

$$
\Delta \epsilon_{t}=180=\epsilon_{b} \Delta \omega
$$

applies, assuming an average $\epsilon_{b}$ can be used. The wall flux change is just the ion flux to the pole piece.

A typical pole piece (Ref. 7 ) $(7.6 \mathrm{~cm}$ diameter and $3.8 \mathrm{~cm}$ in length) and the values $k T_{e} / e=5 \mathrm{eV}$ and $n_{i} / n_{m}=1.1$ will be used. The above equation, along with Eq. (3), results in

$$
\epsilon_{b} n_{i}=8 \times 10^{13}
$$

Assuming a value of $\epsilon_{b}$ of approximately $80 \mathrm{eV}$, an ion density in the pole piece of $10^{12} \mathrm{~cm}^{-3}$ is needed. Densities of this magnitude were found in the 15-cm-diameter thruster [Figs. 2(a) and 3(a)] and could be reasonably expected for the unimproved $20-\mathrm{cm}$-diameter thruster. Therefore, the ion flux to the pole piece computed from reasonable plasma conditions adequately accounts for the change in discharge losses due to a change in cathode position. 
Studies of Ref. 20 showed the discharge losses to be minimized, with respect to cathode position, when the cathode was located at the forward end of the pole piece. The present analysis would predict such an effect. A cathode position within the pole piece adds an ion wall flux. A cathode position beyond the pole piece reduces the "trapping" of primary electrons because the axial magnetic field decreases away from the pole piece. Thus, the best cathode location should be at the forward end of the pole piece.

A difference in discharge losses between oxide and hollow cathode-type thrusters was observed (Ref. 21). The hollow cathode thruster, which normally operates with the cathode at the rear of the pole piece and a baffe, has losses 100 to $200 \mathrm{eV} /$ beam ion higher than the same thruster using an oxide cathode. This increased loss, based on the previous analysis, is attributed to a pole piece ion flux loss.

\section{Screen Grid}

In further tests with the 20-cm-diameter thruster (Ref. 7), a reduction in discharge losses of 250 to $300 \mathrm{eV} /$ beam ion was found by reducing the screen grid thickness $(0.254 \mathrm{~cm}$ to $0.076 \mathrm{~cm}$ ). With this loss reduction, the discharge losses were approximately 170 to $200 \mathrm{eV} /$ ion. This sizable loss reduction can be explained by considering the discussion of Fig. 8 in an earlier section.

As indicated, the screen grid thickness reduction results in an open area increase. In the present case $(20-\mathrm{cm}$ diameter thruster), the open area fraction increase should be approximately $20 \%$ ( 0.60 to 0.72 ). For a given beam current, the increased open area reduces the required ion flux density at the screen. The required ion flux density is reduced by decreasing the total plasma density. As before, the reduced density reduces the ion wall loss $(\omega)$, the collisional loss $\left(\epsilon_{c}\right)$, and the electron energy transport losses $\left(\epsilon_{a}\right.$ and $\left.\epsilon_{h}\right)$ at constant beam current. The reduction in $\epsilon_{b}$ should be approximately $20 \mathrm{eV} /$ ion (i.e., the difference between 80 and $60 \mathrm{eV} /$ plasma ion). A change in $\omega$ of 2.5 to 3.0 is then required to obtain the improved thruster values of $\omega$ near 3.0.

The density reduction resulting from the screen grid modification is expected to produce a larger change in $\omega$ than that for adding the pole piece. The reduced radial drift of electrons caused by the pole piece tends to maintain the cathode and pole piece ion loss, even though the outer wall loss is reduced. However, the general density decrease due to the screen change reduces the cathode, pole piece, and screen grid losses as well as the outer wall losses.

The bulk ion density was apparently reduced more than the $20 \%$ corresponding to the change in screen open area. This might be accounted for by changes in the electron temperature distribution and changes in local ion production rates. Further detailed study of the effects of configuration changes is required to resolve this question.

\section{Propellant Introduction}

Investigations of propellant introduction methods have shown that the "reverse feed" systems (introduction of propellant toward the rear) minimize discharge losses (Ref. 22). In addition, a comparison of "forward" and "reverse" feed methods shows that the maximum propellant utilization efficiency is reduced by approximately 10 to $20 \%$ with the forward system (Ref. 6).

These results are easily interpreted from the previous discussions. At positions greater than approximately half the chamber radius, atoms have mean free paths for ionization of $10 \mathrm{~cm}$ or more. Therefore, atoms leaving the outer portions of the rear surface, or entering through this surface from the propellant system, have a high probability of reaching the grid. Since these atoms can escape directly, a direct loss of propellant in this manner immediately reduces the maximum utilization efficiency obtainable. With a direct loss, achievement of a given utilization efficiency requires a higher ionization fraction resulting in a higher basic ion production cost. With lower plasma density, as in improved thrusters, the forward feed-type system becomes increasingly less efficient.

Directing the propellant toward the rear has an immediate advantage. The directed flux strikes the rear surface and generally evaporates randomly. Thus, only one fourth of the directed propellant flux should go into the axially evaporated flux. This reduces a possible $10 \%$ propellant direct loss, mentioned previously, to a $2.5 \%$ loss. Directing the propellant toward the center as well as toward the rear should result in even less atom axial flux contribution from the original propellant flow. Most atoms passing through the center are ionized. Of those reaching the rear surface, only a small fraction evaporates axially and reaches the grid. This significantly reduces the effect of propellant introduction location.

The effect of propellant introduction configuration (single or multiple entrances) can be considered by evaluating the total mass flowrate off the walls. The ion and 
atom fluxes to the chamber surfaces result in a large evaporated atom flux. This atom flux is equivalent to a "virtual" propellant source with a flowrate $\dot{m}_{0}$ of

$$
\dot{m}_{0}=\frac{m}{e} I_{i w}+\int_{S} \Gamma_{0 w} d S=\frac{m}{e} I_{b}(\omega-1)+\int_{S} \Gamma_{0 w} d S
$$

where $I_{i w}$ is the total ion current to the walls, $I_{b}$ the beam current, $\Gamma_{0 w}$ the flux of atoms to the wall, and $d S$ a unit of wall area. Since

$$
I_{b}=\frac{e}{m} \dot{m} \eta_{m}
$$

Eq. (18) becomes

$$
\dot{m}_{0}=(\omega-1) \eta_{m} \dot{m}+\int_{S} \Gamma_{0 w} d S
$$

For the present calculation, the atom flux will be neglected compared with the ion flux contribution. This is consistent with the use of Eq. (12). For typical improved 20-cmdiameter thruster conditions $\left(\eta_{m}=0.9\right.$ and $\left.\omega=3\right), \dot{m}_{0}=$ $1.8 \dot{m}$. This result shows that the evaporated flux is nearly twice that of the propellant flow. Therefore, the propellant introduction configuration will affect the atom distribution in the plasma only to a small degree.

Therefore, a reverse feed-type propellant system is necessary for achieving high-utilization efficiency with low-discharge losses. However, the location and configuration of propellant introduction using this system is relatively unimportant because of the large atom flux from the walls.

\section{Effects of Operating Conditions}

The variation of discharge performance with operating conditions has a strong influence on the thruster operating range and on control methods (Ref. 7). The observed changes in discharge losses with changes in operating parameters can be discussed by considering $\omega$ and $\epsilon_{b}$. The operating conditions considered here are propellant flowrate, propellant utilization efficiency, discharge voltage, and magnet current.

\section{A. Propellant Flowrate}

Discharge losses are observed to depend directly upon propellant flowrate. The variation is typically 4 to $10 \mathrm{eV} /$ ion per $\mathrm{g} / \mathrm{h}$ of flow. The variation in the improved 20-cm-diameter thruster is near the lower rate (Ref. 7). Over the flowrate range for the 20 -cm-diameter thruster data in Table 2 (5.7 to $9.2 \mathrm{~g} / \mathrm{h}$ ), the total loss change is approximately $16 \mathrm{eV} /$ beam ion. Although this change is not too large in comparison with the changes due to configuration, it represents approximately a $3 \%$ variation in losses per $\mathrm{g} / \mathrm{h}$ of flowrate (for a nominal $150 \mathrm{eV} /$ beam ion).

Propellant flowrate produces an effect similar to that observed in the pole piece and screen grid loss reductions of the previous sections. That is, the flowrate controls the plasma density. This is shown in Figs. 15 and 16 which show ion density and the ratio of primary electron to ion density, respectively. Figure 15, presenting the axial variation of ion density on the centerline, shows that, with increasing flowrate, the density at the cathode increases more rapidly than at the grid. This effect can also be observed

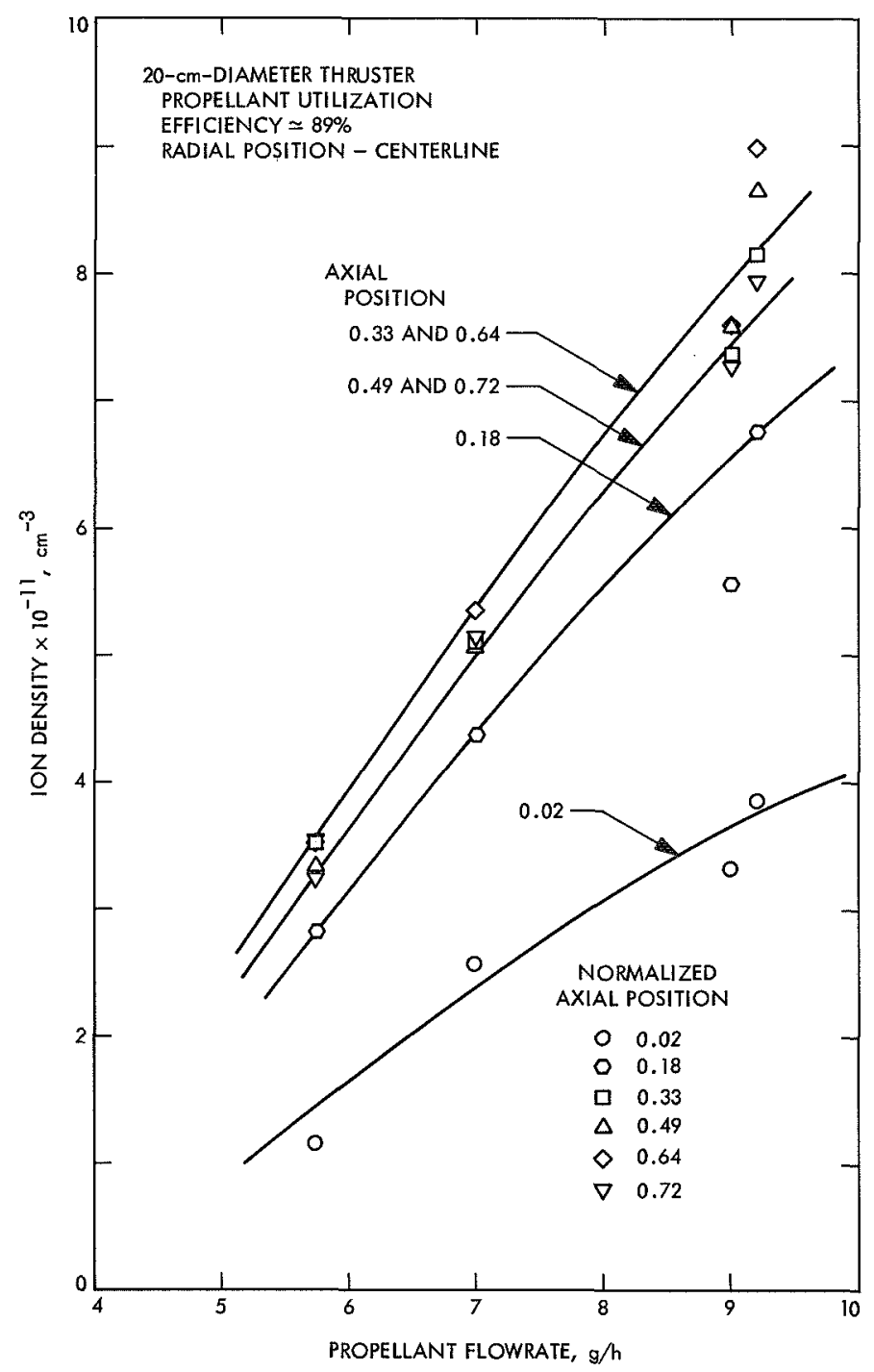

Fig. 15. Ion density variation with propellant flowrate 


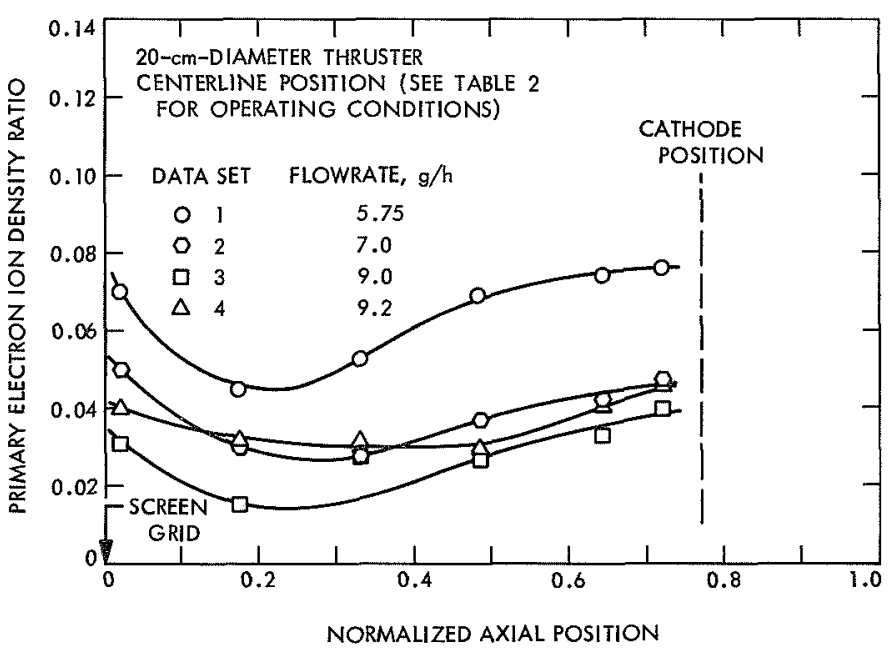

Fig. 16. Ratio of primary electron density to ion density for four flowrates

in Figs. 4(a) and 6(a). The increased density at the cathode, relative to that at the grid, results in an increase in $\omega$ with flowrate. This can be observed from the data in Table 2 which shows that the increase in $\omega$ with flowrate (2.87 to 3.08 ) occurs principally in the cathode flux contribution.

Although the ratio of primary electrons to ions changes with flowrate (Fig. 16), the basic production cost is not significantly affected. This is illustrated by the relatively constant values of $\epsilon_{b}$ in Table 2. As shown previously, the Maxwellian and primary electrons contribute approximately equally to ionization and with approximately equal cost. Therefore, a tradeoff of Maxwellian electrons for primary electrons in the present case results in little change in $\epsilon_{b}$.

\section{B. Propellant Utilization Efficiency}

The variation of discharge losses with propellant utilization plays a key role in determining the thruster operating point. A plot of discharge loss as a function of utilization, as shown in Fig. 17, shows that the losses increase with increased utilization. It should be noted that the utilization in Ref. 7 was adjusted by means of the discharge current. Adjustment of utilization by use of the discharge voltage, as in Ref. 23, produces somewhat different discharge loss-utilization curves. This difference appears to result from use of low discharge voltages in the latter tests. There are three principal characteristics of the discharge loss-utilization curve presented in Fig. 17. At low utilization, the curve slope is rather low ( 5 to $10 \mathrm{eV} /$ beam ion per $10 \%$ change in utilization). At high utilization, the curve

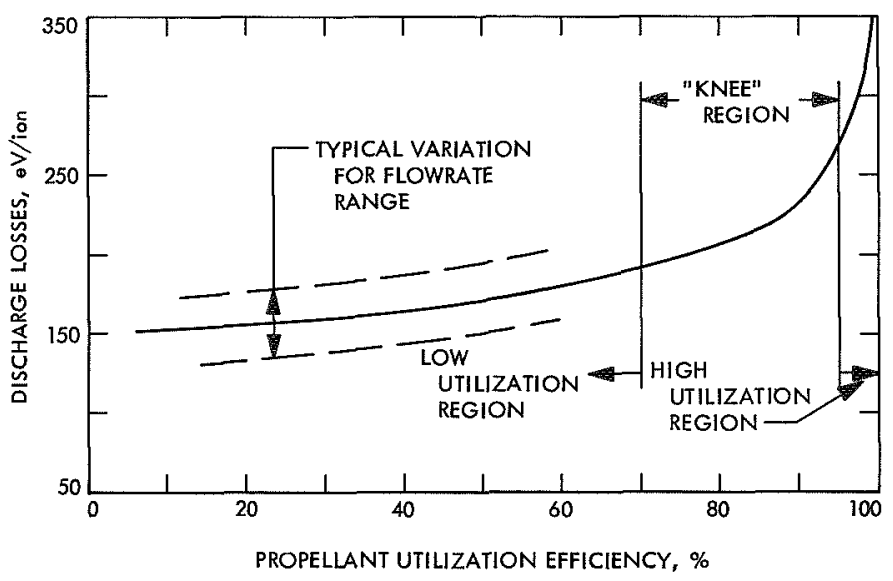

Fig. 17. Typical discharge loss-propellant utilization efficiency curve

slope is high (approaching infinity at $100 \%$ utilization). Between these regions is a transition or "knee." The total thruster efficiency depends on both utilization efficiency and power efficiency, which is strongly coupled to the discharge loss. Therefore, a tradeoff of discharge power against utilization must be made in the "knee" region to achieve maximum thruster efficiency. It is of interest to consider the plasma characteristics that produce this curve shape.

All plasma properties were found to change with utilization. Ion density and primary electron density are shown in Fig. 18 as a function of utilization. The data were

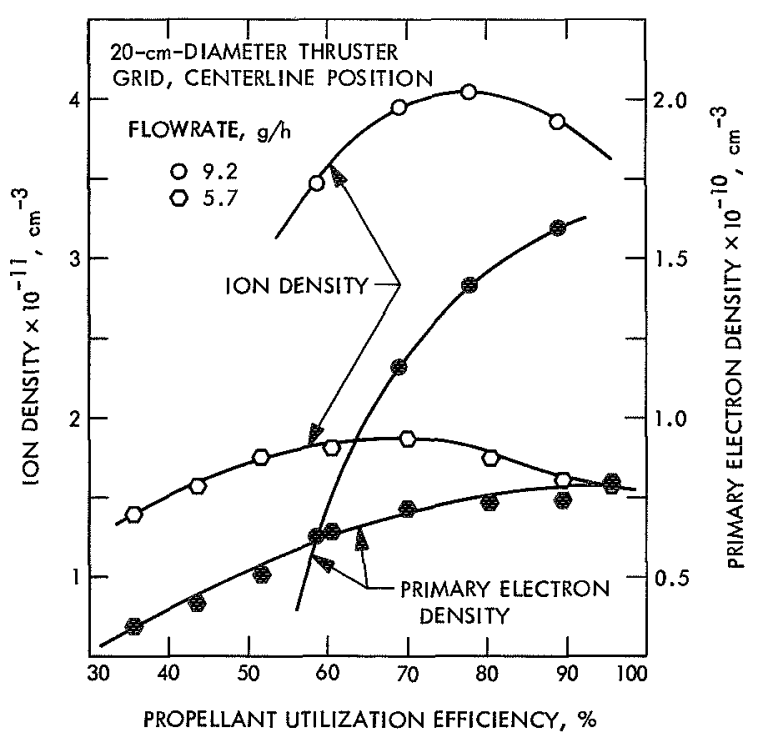

Fig. 18. Ion density variation with propellant utilization efficiency 
taken on the centerline near the screen grid in the $20-\mathrm{cm}$ diameter thruster. The radial variation of ion density is shown for the two thrusters in Fig. 19(a), for the 15-cmdiameter thruster, and Fig. 19(b), for the 20-cm-diameter thruster. In these, utilization efficiency is a parameter. Data for two axial positions are given for the $15-\mathrm{cm}$ diameter thruster. An increase in primary electron density with utilization could be expected because the discharge current increases with utilization. The variation of ion density with utilization, showing a maximum at 70 to $80 \%$, will be shown to be related to changes in Maxwellian electron temperature. Maxwellian electron temperature and primary electron energy (for the 20 -cm-diameter thruster only) are shown in Fig. 20 for the two thrusters. These data show the strong dependence of electron energy on utilization. The radial distribution of Maxwellian electron temperature for the two thrusters is shown in Fig. 21 for variable utilization.

In the low utilization region, the ion density and electron wall flux (discharge current) are low. This condition minimizes, with respect to density, the collisional loss and the
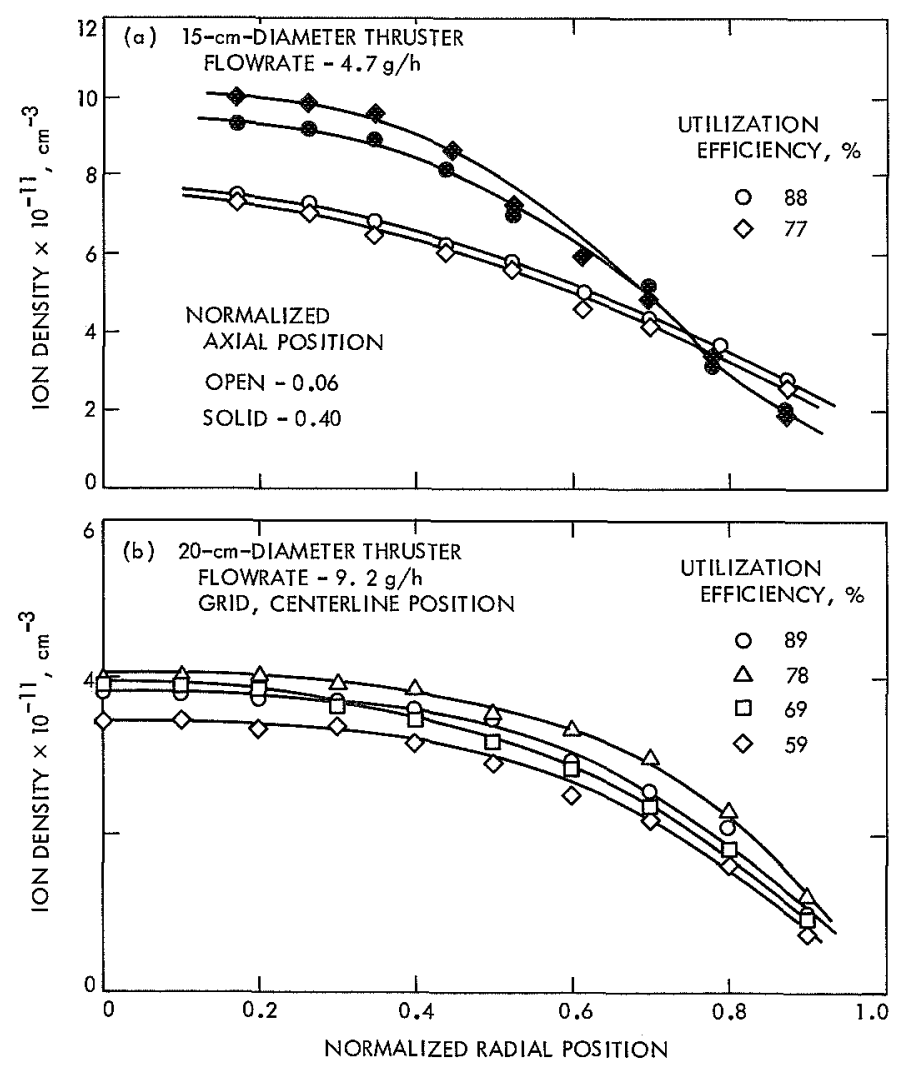

Fig. 19. Ion density radial variation as a function of propellant utilization efficiency electron energy transport loss. However, the electron energy is low (Fig. 20). An increase in utilization increases the ion density, discharge current, and electron energy. The increase in electron energy compensates in $\epsilon_{c}^{\prime} \dot{v}_{i}$ for the density increase. Hence, the collisional loss tends to remain constant. The wall loss factor $\omega$ and the electron energy transport losses, $\epsilon_{h}$ and $\epsilon_{a}$, increase with utilization due to the increased density. Therefore, in the low-utilization region, the discharge loss changes depend on changes in $\omega, \epsilon_{h}$, and $\epsilon_{a}$. The improvement study of Ref. 7 showed that the slope of the discharge loss utilization curve decreased as improvements were made. This reduced slope corresponds to the reduced influence of changes in $\epsilon_{h}$ and $\epsilon_{a}$ as these quantities were reduced (Table 4).

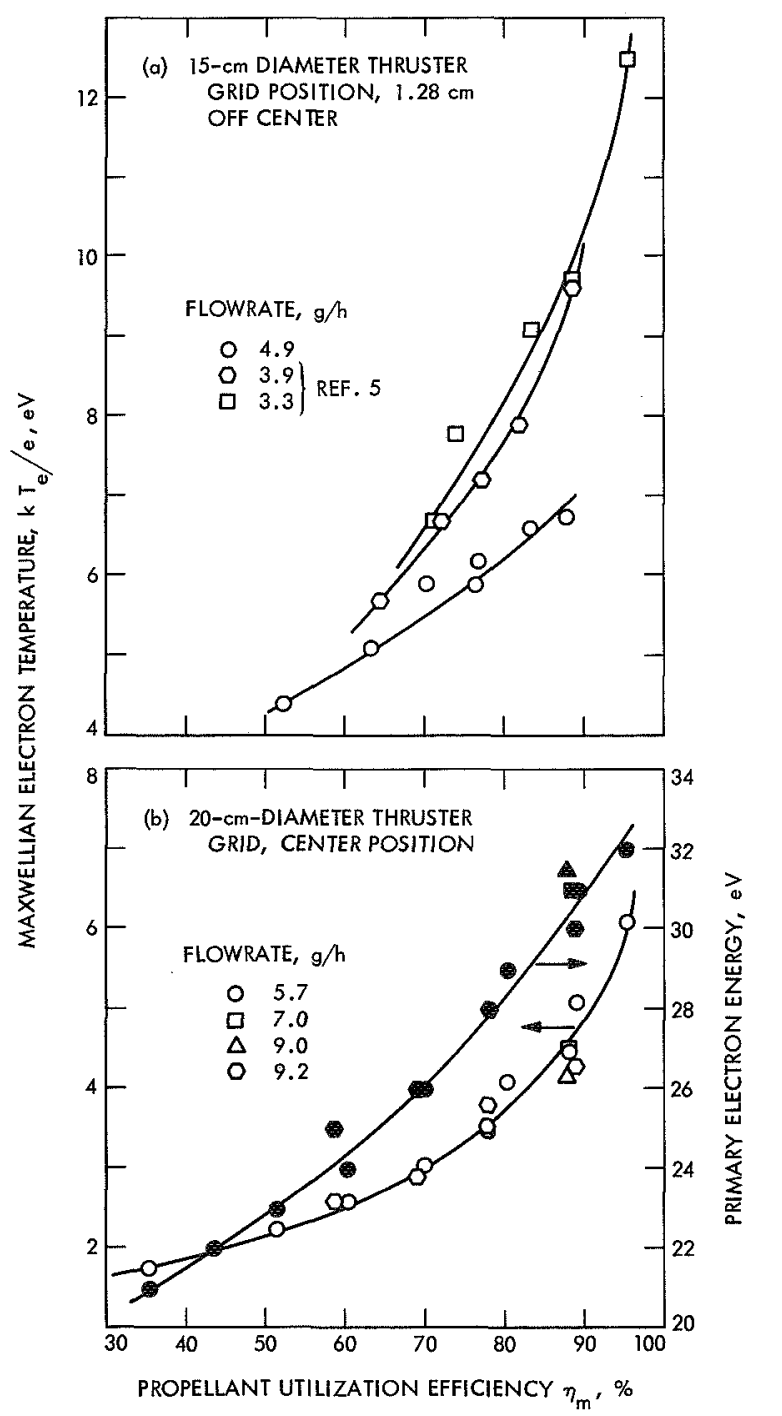

Fig. 20. Maxwellian electron femperature variation with propellant utilization efficiency 

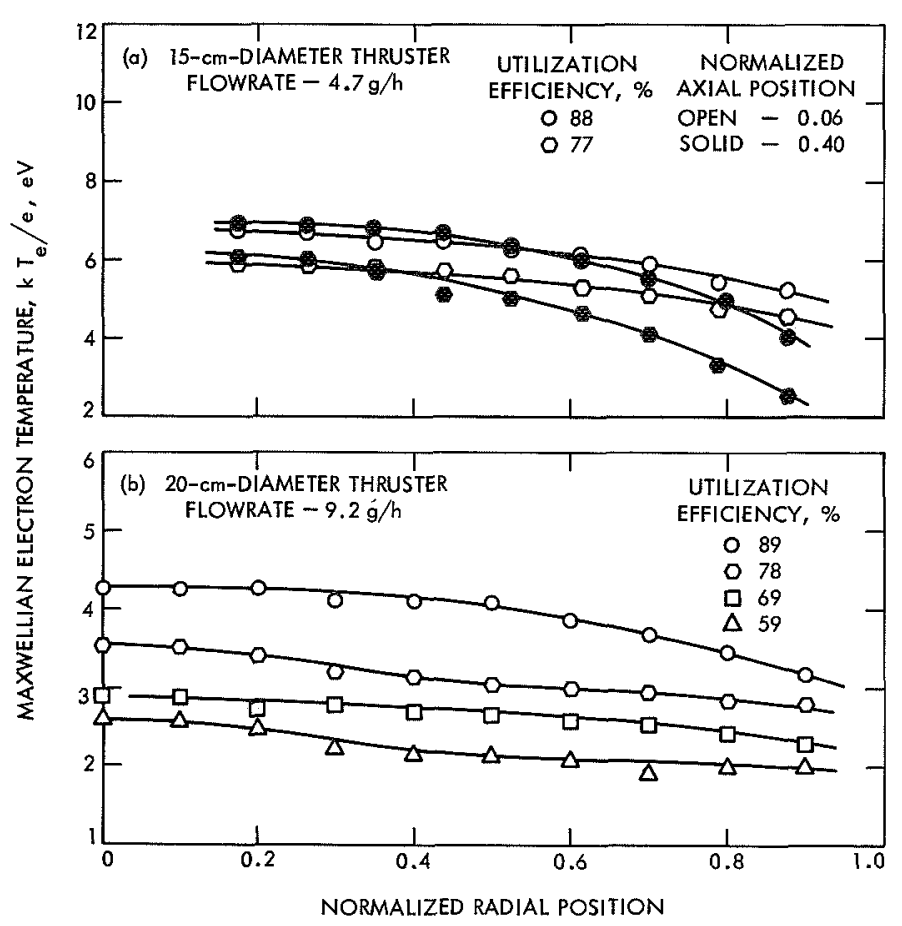

Fig. 21. Maxwellian electron temperature radial variation as a function of propellant utilization efficiency

In the "knee" region (70 to $95 \%$ ) of the discharge loss curve, electron energy continues to increase, but ion density reaches a maximum and begins to decrease. This decrease in ion density is made possible by the rapid increase in electron temperature. The increased temperature increases the ion flux according to the Bohm criterion (Eq. 3) and, hence, increases the beam current. Similarly, the increased temperature increases $\omega, \epsilon_{h}$, and $\epsilon_{a}$ by increasing the ion and electron wall fluxes.

For a given flowrate, an increase in utilization also requires a decrease in atom flux from the thruster. Most of the escaping atoms originate from the anode or the outer area of the rear surface. To reduce this flux, the mean free path for ionization of an atom $\lambda_{0 e}^{*}$ for the outer plasma regions must decrease with utilization. As shown in Eq. (11), $\lambda_{0 e}^{*}$ depends on electron density and temperature $\left(\Sigma=\Sigma\left(T_{e}\right)\right)$. The electron density (except in the grid region) and electron temperature are increased to accomplish the reduction in $\lambda_{\mathrm{o} e}^{*}$. However, these increases result in higher wall losses and higher collisional losses. In addition, the increased ion wall flux produces a higher atom flux. Therefore, the density and temperature, and hence the losses, increase rapidly at high utilization.

The location of the "knee" can be expected to depend strongly upon the atom flux distribution (from the walls or from introduction). Propellant injected toward the grid, as with "forward" feed-type propellant systems, will effectively shift the discharge loss curve toward lower utilization. Since this "direct" loss can be overcome by reducing $\lambda_{0 e}^{*}$ through temperature increases, the discharge loss curve begins increasing at a lower utilization. This tends to make the curve more rounded.

A good example of this effect is the present hollow cathode thruster. A portion of the propellant injected through the hollow cathode enters the discharge unionized. Since this atom flux is directed axially, a direct propellant loss is possible. It should be noted that $\lambda_{0 e}^{*}$ is on the order of $5 \mathrm{~cm}$ in the central plasma region. Considering the exponential atom flux decay, approximately $8 \%$ of a directed flux leaving the pole piece could reach the grid unionized. The hollow cathode, in the present configuration (allowing an axial atom flux), has a more rounded discharge loss curve than the equivalent thruster using an oxide cathode (Ref. 7).

\section{Discharge Voliage}

Thruster tests show that discharge voltages in the range of 30 to $50 \mathrm{~V}$ do not significantly affect discharge performance (Ref. 7). Voltages below $30 \mathrm{~V}$ increase the losses. Those above $50 \mathrm{~V}$ appear to produce a significant fraction of doubly ionized ions and distort the test results (Ref. 1). Voltages above $50 \mathrm{~V}$ are not commonly used and will not be considered here. The effect of discharge voltages below $40 \mathrm{~V}$ will be discussed as before in terms of $\omega$ and $\epsilon_{b}$.

The variation of ion and primary electron density with discharge voltage for the 20-cm-diameter thruster is shown in Fig. 22(a). The corresponding Maxwellian electron temperature and primary electron energy are given in Fig. 22(b). These data were taken at constant utilization efficiency at the center grid position.

A decrease in Maxwellian electron temperature with increasing voltage is indicated by the data. This results from the fact that the primary electron current is decreased with voltage. Therefore, fewer primary electrons are available to maintain the Maxwellian temperature. As the Maxwellian temperature decreases, the collisional loss $\epsilon_{c}^{\prime}$ increases because the change in the primary electron contribution to this loss is small (Fig. 13). However, the temperature decrease reduces $\omega$. The changes in $\epsilon_{b}$ (from $\epsilon_{c}$ ) appear to be balanced by the changes in $\omega$ resulting in a near-constant total loss $\epsilon_{t}$.

The decrease in ion density at higher discharge voltages is not explained by the present data. However, the 

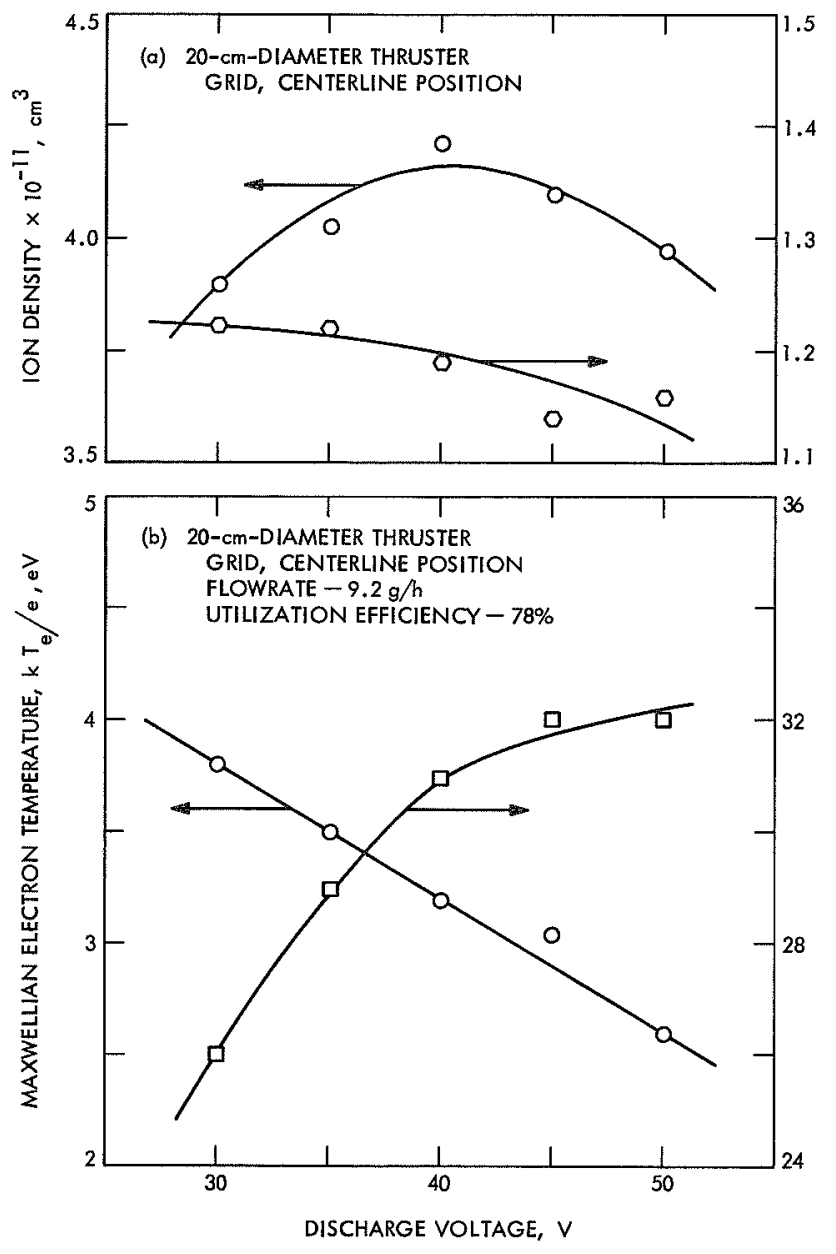

Fig. 22. Ion density and Maxwellian electron temperature variation with discharge voltage

change in ion density at the center with voltage, at constant beam current, must correspond to a change in the ion density radial distribution.

\section{Magnet Current}

The effect of magnet current (or axial magnetic field strength) on discharge losses was shown in Ref. 7. At low magnet current, the losses are inversely proportional to current and the discharge loss-utilization curve has a relatively steep slope. As the current increases, the losses reach a minimum and begin increasing. Further magnet current increases often cause the discharge to become unstable. The analysis of this variation in losses follows from plasma data.

Radial distributions of ion density and Maxwellian electron temperature at the grid are shown in Fig. 23. These data were obtained at constant utilization in the $20-\mathrm{cm}$ diameter thruster.
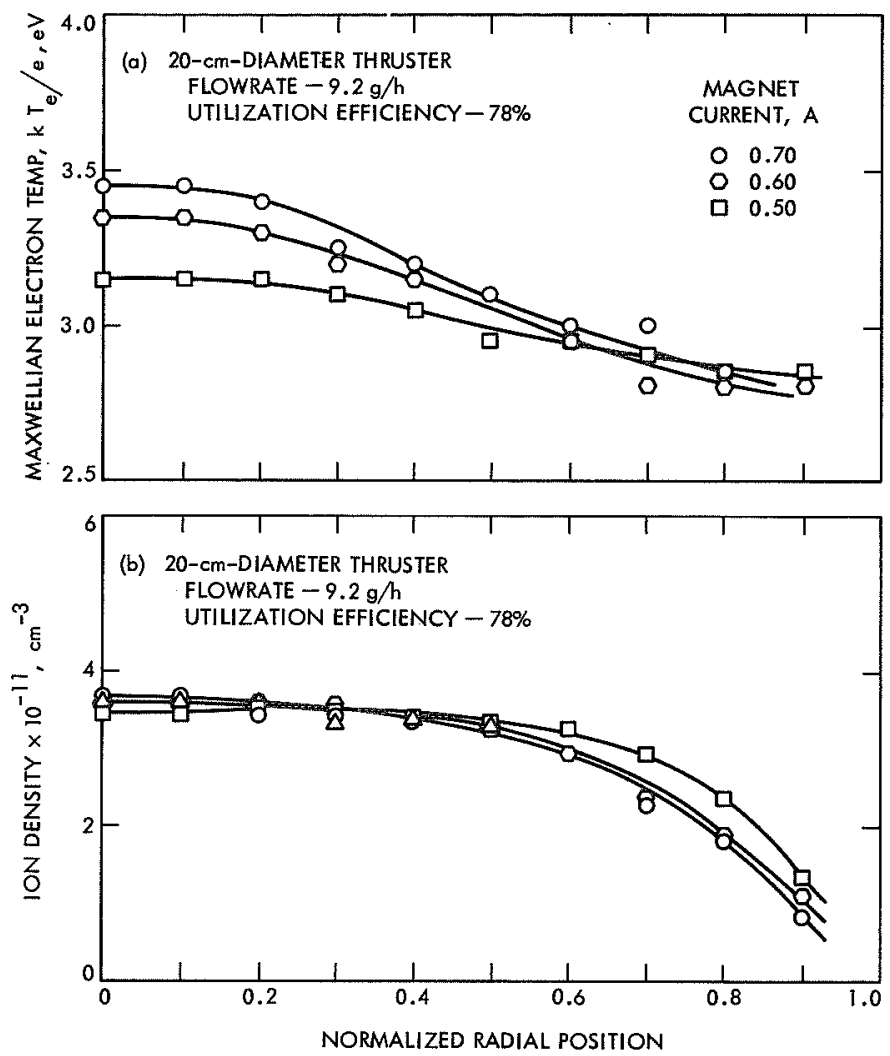

Fig. 23. Ion density and Maxwellian electron temperafure variation with magnet current

For increasing magnet current, the electron temperature increases in the center but remains relatively constant near the wall. The ion density remains constant in the center but decreases at the wall. These distribution changes result from an effect similar to that found by adding a pole piece. An increased magnetic field forces primary electrons to deposit relatively more energy in the grid region. The increase in Maxwellian temperature shows this effect. At constant utilization, the beam ion flux is constant and the ion density is adjusted by the plasma potential to supply this current. Since the Maxwellian temperature has increased in the center, requiring a higher ion flux, the ion density must decrease in the outer region.

For low field operation, the ion wall loss is reduced by an increase in magnet current. This occurs because the ion density at the wall decreases while the Maxwellian temperature remains relatively constant. As the magnet current is increased further, the temperature distribution becomes more nonuniform. The ion density increases in the central region to supply the beam ion flux required at the center. This increases $\epsilon_{c}^{\prime}$ in this region and compensates for the reduced wall loss. This explains the fact 
that the discharge loss-magnet current curve reaches a minimum and can increase with higher magnet current (higher $\epsilon_{c}^{\prime}$ ).

The grid or ion optics system producing the beam is limited to a maximum current density. Ion beam currents greater than this value result in direct ion impingement on the accelerator grid. Increased magnet current, as indicated, results in increased current density in the center. Therefore, even at constant total beam current (or constant utilization), the magnet can produce high or unstable accelerator impingement. An example of this condition is shown in Ref. 6. If this impingement limit is reached, higher total beam current could be drawn by reducing the magnet current. This effect was also observed in the investigation leading to Ref. 7 .

If the impingement limit is not encountered, an additional difficulty may occur. A continued increase in magnetic field could limit electron transport to the anode. The reduced transport is indicated by the reductions shown in Fig. 22. This would reduce or limit the discharge current and could extinguish the discharge. In general, the impingement limit is reached first in the bombardment thruster.

\section{Condusions}

The analysis presented in this report was successful in establishing the basic relationships between the plasma properties and thruster discharge performance. A number of conclusions can be drawn from this work, as follows:

(1) The ion velocity determined by the modified Bohm criterion and applied at the screen grid sheath, produces the measured beam current. This result was used to compute total ion flux to the beam and walls. An independent calculation of the total ion flux, using the total ion production rate, verified the Bohm calculation.

(2) The principal plasma losses, contributing to the ion production cost per plasma ion $\epsilon_{b}$, were shown to be inelastic collisional losses (resulting in excitation and subsequent radiation) and electron energy transport to the anode. In present thrusters, the collisional losses (atoms and ions) contribute approximately $90 \%$ of the total loss. A lower limit on the value of $\epsilon_{b}$ can be made from this result. The lower limit on the collisional loss (Fig. 13) appears to be approximately $20 \mathrm{eV} /$ plasma ion. Therefore, considering the housing and anode losses, a limiting value for $\epsilon_{b}$ of approximately $30 \mathrm{eV} /$ plasma ion can be expected. However, this low basic cost may not be consistent with a low ion wall loss factor since a high electron energy is required for low $\epsilon_{b}$. Therefore, a value of $40 \mathrm{eV} / \mathrm{plasma}$ ion is a more realistic estimate for the achievable limit on $\epsilon_{b}$.

(3) Additional reductions in the ion wall loss factor seem possible. Increases in screen grid open area fraction should be the most direct approach. Obvious mechanical difficulties restrict this possible improvement. Direct insulation of the housing surfaces, or ion trapping (by use of magnetic fields as demonstrated by Moore in Ref. 24), may reduce the wall loss fraction. Values of $\omega$ less than approximately 1.5 seem unlikely. In this case, a lower limit on total discharge losses of approximately $60 \mathrm{eV}$ /beam ion seems probable. However, for conventional thrusters without ion trapping, a limit on () of approximately 2 is expected. This indicates a lower limit on discharge losses of approximately $80 \mathrm{eV} /$ beam ion for the conventional thruster.

(4) The higher discharge losses observed using a hollow cathode, compared to an oxide cathode, are directly related to ion losses to the interior surfaces of the pole piece. Reduction of this surface area, by insulation or size reduction, is expected to improve hollow cathode thruster performance.

(5) Comparison of the basic losses for mercury and cesium thrusters, using data from Ref. 1 for cesium thrusters and Ref. 17 for $\epsilon_{c}^{\prime}$, shows little difference between these propellants. It is noted that, although ionization potentials are quite different for cesium and mercury, the electron temperature in the plasma is proportional to the ionization potential. Therefore, a direct comparison of propellants for the present thruster must consider plasma properties as well as the physical properties of the propellant. 


\section{Appendix}

\section{Modified Bohm Criterion}

The presence of primary electrons in the plasma requires the Bohm stable sheath analysis (Ref. 14) to be modified. As initially derived for a Maxwellian velocity distribution, the Bohm criterion states that

$$
\epsilon_{i} \supseteq \frac{k T_{e}}{2 e}
$$

where $\epsilon_{i}$ is the mean energy of ions approaching the sheath with velocity normal to the sheath. The following analysis includes the contribution of primary electrons to the velocity distribution function.

The Poisson equation

$$
\frac{\partial^{2} V}{\partial x^{2}}=4 \pi e\left[n_{i}(x)-n_{e}(x)\right]
$$

is considered in the sheath region. The potential in the sheath, $V$, is taken to be zero at the plasma-sheath boundary. That is, $V=0$ at $x=0$. The electron density in the sheath $n_{e}(x)$ is given by

$n_{e}(x)=n_{m}(0) e^{-\mathrm{eV}(x) / k T_{e}}+n_{p}(0)\left(1-\frac{V(x)}{\epsilon_{p}}\right)$

where $n_{m}(0)$ and $n_{p}(0)$ are the Maxwellian and primary electron densities at the sheath boundary respectively and $\epsilon_{p}$ is the primary electron energy in $\mathrm{eV}$. The second term in Eq. (A-3) accounts for a monoenergetic electron contribution to the distribution function. The ion density, as in the original analysis (Ref. 14), is given by

$$
n_{i}=n_{i}(0)\left(\frac{\epsilon_{i}}{\epsilon_{i}+V}\right)^{1 / 2}
$$

Substituting Eqs. (A-3) and (A-4) into Eq. (A-2)

$$
\begin{aligned}
\frac{\partial^{2} V}{\partial x^{2}}= & 4 \pi e n_{i}(0)\left[\left(\frac{\epsilon_{i}}{\epsilon_{i}+V}\right)^{1 / 2}\right. \\
& \left.-\frac{n_{m}(0)}{n_{i}(0)} e^{-\mathrm{eV} / k T_{e}}-\frac{n_{p}(0)}{n_{i}(0)}\left(1-\frac{V}{\epsilon_{p}}\right)\right]
\end{aligned}
$$

Multiplying Eq. (A-5) by $\partial V / \partial x$ and integrating results in the equation

$$
\begin{aligned}
\left(\frac{\partial V}{\partial x}\right)^{2}= & 8 \pi n_{i}(0) e\left[2 \epsilon_{i}^{1 / 2}\left(\epsilon_{i}+V\right)^{1 / 2}\right. \\
& \left.+\frac{n_{m}(0)}{n_{i}(0)}\left(\frac{k T_{e}}{e}\right) e^{-\mathrm{eV} / k T_{e}}+\frac{n_{p}(0)}{n_{i}(0)}\left(\frac{V^{2}}{\epsilon_{p}}-V\right)\right]+C
\end{aligned}
$$

Applying the boundary condition

$$
\frac{\partial V}{\partial x}=0 \quad \text { at } \quad V=0
$$

the constant $C$ can be evaluated. This leads to

$$
\begin{aligned}
\left(\frac{\partial V}{\partial x}\right)^{2}= & 8 \pi n_{i}(0) e\left[2 \epsilon_{i}^{1 / 2}\left(\epsilon_{i}+V\right)^{1 / 2}-2 \epsilon_{i}\right. \\
& \left.+\frac{n_{m}(0)}{n_{i}(0)}\left(\frac{k T_{e}}{e}\right)\left(e^{-\mathrm{eV} / k T_{e}}-1\right)+\frac{n_{p}(0)}{n_{i}(0)}\left(\frac{V^{2}}{\epsilon_{p}}-V\right)\right]
\end{aligned}
$$

Near the plasma-sheath boundary

$$
\begin{aligned}
& V<<\frac{k T_{e}}{e}<<\epsilon_{p} \\
& V<<\epsilon_{i}
\end{aligned}
$$

The term $\left(\epsilon_{i}+V\right)^{1 / 2}$ and the exponential can be expanded in series to reduce Eq. (A-6) to the form

$$
\left(\frac{\partial V}{\partial x}\right)^{2}=4 \pi n_{i}(0) e\left(\frac{n_{m}(0)}{n_{i}(0)} \frac{e}{k T_{e}}-\frac{1}{2 \epsilon_{i}}\right) V^{2}
$$

The left side of Eq. (A-7) is always positive, requiring

$$
\frac{n_{m}(0)}{n_{i}(0)} \frac{e}{k T_{e}} \supseteq \frac{1}{2 \epsilon_{i}}
$$

for a real solution of Eq. (A-7) (i.e., a stable sheath). In terms of the ion energy

$$
\epsilon_{i} \supseteq\left(\frac{k T_{e}}{\partial e}\right)\left(\frac{n_{i}(0)}{n_{m}(0)}\right)
$$

with a primary electron density at the sheath, $n_{i}(0) / n_{m}(0)$ is greater than unity. Therefore, the primary electrons increase the ion energy or velocity required to maintain a stable sheath. 


\section{Nomenclature}

$A_{j}$ area segment

$c_{e} \quad\left(2 k T_{e} / m_{e}\right)^{1 / 2}$

$e$ electronic charge

$G$ defined after Eq. (12)

$I_{\alpha} \quad$ current of species $\alpha$

$I_{b}$ beam current

$k$ Boltzman constant

$L$ thruster chamber length

$m_{\alpha}$ particle mass of species $\alpha$

$\dot{m}$ flowrate

$n_{\alpha} \quad$ particle density of species $\alpha$

$q_{\alpha}$ energy flux of species $\alpha$

$Q$ cross section (see Eq. 13)

$r$ radial coordinate

$R$ anode radius

$T_{a} \quad$ temperature of species $\alpha$

$v_{\alpha}$ particle velocity of species $\alpha$

$v_{s}$ electron velocity in a direction normal to the sheath

$V$ energy level (see Eq. 13)

$V_{s}$ sheath potential

$x$ sheath thickness

$\mathrm{Z}$ axial coordinate

$\alpha$ defined after Eq. (9)

$\beta$ defined after Eq. (15)

$\Gamma_{\alpha}$ particle flux of species $\alpha$ $\epsilon_{a}$ particle energy of species $\alpha$

$\epsilon_{a}$ anode energy loss

$\epsilon_{b}$ basic ion production cost

$\epsilon_{c} \quad$ average collisional energy loss

$\epsilon_{c}^{\prime} \quad$ local collisional energy loss

$\epsilon_{h}$ housing energy loss

$\epsilon_{t} \quad$ defined by Eq. (2)

$\epsilon_{0}$ permittivity of vacuum

$\eta_{m}$ propellant utilization efficiency

$\lambda_{0 e}^{*} \quad$ atom mean free path for ionization

$\lambda$ average atom mean free path defined after Eq. (15)

$\dot{v}_{i}$ local ion production rate

$\Sigma_{\alpha} \quad$ coefficient for ionization by species $\alpha$

$\phi_{i} \quad$ effective grid open area for ions

$\phi_{0} \quad$ effective grid open area for atoms

$\omega$ ratio of total ion wall flux to beam flux

Subscripts

$\boldsymbol{e}$ electron

$i$ ion or ionization potential

$j$ atomic energy level

$k$ ionic energy level

$m$ Maxwellian electron

0 atom

$p$ primary electron

\section{References}

1. Kohlberg, I., and Nablo, S., "Physical Phenomena in Bombardment Ion Sources," in Physics and Technology of Ion Motors, Edited by Frank E. Marble and Jean Surugue, Gordon and Breach Science Publishers, 1966.

2. Masek, T. D., Plasma Characteristics of Electron Bombardment Ion Engines, Technical Report 32-1271. Jet Propulsion Laboratory, Pasadena, Calif., April 15, 1968. (Also presented at the 2nd International Conference on Electron and Ion Beam Science and Technology, New York, April 1966.) 


\section{References (contd)}

3. Kaufman, H. R., Performance Correlation for Electron-Bombardment Ion Sources, TN D-3041, National Aeronautics and Space Administration, October 1965.

4. Strickfaden, W. B., and Geiler, K. L., Probe Measurements of the Discharge in an Operating Electron Bombardment Engine, Technical Report 32-417. Jet Propulsion Laboratory, Pasadena, California, April 19, 1964. (Also AIAA J., Vol. 1, 1963, pp. 1815-1823.)

5. Masek, T. D., Plasma Studies in the Electron Bombardment Ion Engine, Space Programs Summary 37-42, Vol. IV, November 30, 1965, and Space Programs Summary 37-44, Vol. IV. Jet Propulsion Laboratory, Pasadena, Calif., March $31,1966$.

6. Masek, T. D., and Womack, J. R., "Experimental Studies with a Mercury Bombardment Ion Engine System," AIAA Paper 67-698, AIAA Electric Propulsion and Plasma Dynamics Conference, Colorado Springs, Colorado, September 1967. (Also Technical Report 32-1280, Jet Propulsion Laboratory, Pasadena, Calif., July 15, 1968)

7. Masek, T. D., and Pawlik, E. V., "Thrust System Technology for Solar Electric Propulsion," AIAA Paper 68-541, presented at the 4th Propulsion Joint Specialist Conference, Cleveland, Ohio, June 1968.

8. Kerrisk, D. J., and Masek, T. D., "Effects of Plasma Non-Uniformity on Grid Erosion in an Electron Bombardment Ion Engine," AIAA J., Vol. 3, 1965, pp. 1060-1066. (Also Technical Report 32-727, Jet Propulsion Laboratory, Pasadena, Calif., July 30, 1965.)

9. Masek, T. D., Plasma Investigations in the SE-20C Thruster, Space Programs Summary 37-53, Vol. III. Jet Propulsion Laboratory, Pasadena, Calif., October 31, 1968.

10. Langmuir, I., "The Interaction of Electron and Positive Ion Space Charges in Cathode Sheaths," Physics Review, 31, 954 (1929).

11. Mitchell, A. C. G., and Zemansky, M. W., Resonance Radiation and Excited Atoms, Cambridge University Press, 1961.

12. Sutton, G. W., and Sherman, A., Engineering Magnetohydrodynamics, McGrawHill Book Co., Inc., 1965.

13. Rapp, D., and Francis, W. E., "Charge Exchange Between Gaseous Ions and Atoms," J. Chem. Phys., 37, 2631-2645 (1962).

14. Bohm, D., "Minimum Ionic Kinetic Energy for a Stable Sheath," The Characteristics of Electrical Discharges in Magnetic Fields, A. Guthrie and R. K. Wakerling, eds., McGraw-Hill Book Co., Inc., 1949, pp. 77-86.

15. Brewer, G. R., et al., Ion Engine Thrust Vector Study, Hughes Research Laboratories, Quarterly Report No. 1, Phase II, Jet Propulsion Laboratory Contract No. 952129, December 1968.

16. Kerrisk, D. J., Arc-Type Ion Sources for Electrical Propulsion, Technical Note 61-4, Aeronautical Systems Division, Wright-Patterson Air Force Base, May 1961. 


\section{References (contd)}

17. Dugan, J. V., and Souie, R. J., Volume Ion Production Costs in Tenuous Plasmas, TN 4-4150, National Aeronautics and Space Administration, September 1967.

18. Arnot, F. L., and Baines, G. O., "Elastic and Inelastic Cross Sections of the Mercury Atom," Proc. Roy. Soc., A151, 256-274 (1935).

19. Handbook of Chemistry and Physics, The Chemical Rubber Publishing Co., Cleveland, Ohio, 1962.

20. Bechtel, R. T., "Discharge Chamber Optimization of the SERT II Thruster," AIAA Paper 67-668, AIAA Electric Propulsion and Plasmadynamics Conference, Colorado Springs, Colo., September 1967.

21. Masek, T. D., and Pawlik, E. V., Hollow Cathode Operation in the SE-20C Thruster, Space Programs Summary 37-53, Vol. III. Jet Propulsion Laboratory, Pasadena, Calif., October 31, 1968.

22. Reader, Paul D., Experimental Effects of Propellant-Introduction Mode on Electron-Bombardment Ion Rocket Performance, TN D-2587, National Aeronautics and Space Administration, 1965.

23. Knauer, W., et al., Discharge Chamber Studies for Mercury Bombardment Ion Thrusters, Hughes Research Laboratories, NASA CR-72440, September 1968.

24. Moore, D. R., "Magneto-Electrostatic Bounded Plasma Ion Thruster," Paper presented at the AIAA 7th Electric Propulsion Conference, Williamsburg, Va., March 3, 1969. 